\title{
Reduced power operations in motivic cohomology $\mathbf{y}^{1,2}$ \\ by Vladimir Voevodsky
}

May 2001

\section{Contents}

1 Introduction . . . . . . . . . . . . . . . . 1

2 Motivic cohomology and operations . . . . . . . . . 3

3 Operations $\tilde{H}^{2 d, d} \rightarrow \tilde{H}^{2 d+*, d+i}$ for $i \leq 0 \ldots \ldots$

4 Thom isomorphism and Euler classes . . . . . . . . . . . 12

5 Total power operations . . . . . . . . . . . . . . . 14

$6 \quad$ Motivic cohomology of $B \mu_{l}$ and $B S_{l} \ldots \ldots \ldots$

7 Symmetry theorem . . . . . . . . . . . . . . 27

8 Power operations and the Bockstein homomorphism . . . . . . 31

9 Individual power operations: formulas . . . . . . . . . . . . . 33

10 Adem relations . . . . . . . . . . . . . . . . 37

11 Motivic Steenrod algebra . . . . . . . . . . . . . . 42

12 Structure of the dual to the motivic Steenrod algebra . . . . . 45

13 Operations $\rho(E, R)$ and their properties . . . . . . 53

14 Operations and characteristic classes . . . . . . . . 56

\section{Introduction}

In this paper we consider cohomological operations in the motivic cohomology of smooth simplicial schemes over a field $k$. For the most part we work with cohomology with coefficients in $\mathbf{Z} / l$ where $l$ is a prime different from the characteristic of $k$. We construct the reduced power operations

$$
P^{i}: H^{*, *}(-, \mathbf{Z} / l) \rightarrow H^{*+2 i(l-1), *+i(l-1)}(-, \mathbf{Z} / l)
$$

\footnotetext{
${ }^{1}$ Supported by the NSF grants DMS-97-29992 and DMS-9901219 and The Ambrose Monell Foundation

${ }^{2}$ School of Mathematics, Institute for Advanced Study, Princeton NJ, USA. e-mail: vladimir@ias.edu
} 
and prove the motivic analogs of the Cartan formula and the Adem relations. We also describe the subalgebra in the algebra of all (bistable) operations in the motivic cohomology with $\mathbf{Z} / l$-coefficients generated by operations $P^{i}$, the Bockstein homomorphism and the multiplication by the motivic cohomology classes of $\operatorname{Spec}(k)$. For odd $l$ this algebra is isomorphic to the twisted tensor product of the usual topological Steenrod algebra with the motivic cohomology ring of the point $H^{*, *}$ with respect to the action of the motivic Steenrod operations on $H^{*, *}$. For $l=2$, the situation is more complicated since the motivic Adem relations involve nontrivial coefficients from $H^{*, *}$.

To construct the reduced power operations we follow the approach of [7] where one first defines the total power operation and then uses the computation of the cohomology of the product of a space with the classifying space of the symmetric group $S_{l}$ to obtain the individual power operations. We also use the ideas of [7] for the proofs of the relations between power operations and the Bockstein homomorphism, the Cartan formula and the Adem relations. Our construction of the total power operations is not directly similar to any of the standard topological constructions ${ }^{3}$. One would get its direct topological analog if one unfolded, to the space-level, the description of power operations in terms of $E_{\infty}$-structure on the Eilenberg-MacLane spectra. In particular, the Thom isomorphism in the motivic cohomology and the Euler classes of vector bundles figure prominently in most of our computations.

Several important results on operations in motivic cohomology are not discussed in this paper and will be proved in a sequel. We do not show that the operations $P^{i}$ are unique. We do not show that the operations $P^{i}$ and the Bockstein homomorphism generate all bistable cohomological operations. Finally, we do not prove that bistable cohomological operations coincide with the endomorphisms of the Eilenberg-Maclane spectrum in the stable category.

Modulo this identification of bistable operations with the endomorphisms of $H_{\mathbf{z} / l}$, the present paper contains proofs of the following results used in [9] for the proof of the Milnor conjecture: [9, Theorem 3.14 p.31] is Propositions 3.7 and 3.8; [9, Theorem 3.16 p.32] is Theorem 9.4, Lemma 9.7, Proposition 9.6 and Lemma 9.5. As Example 13.7 shows, the inductive construction used in [9] to define the operations $Q_{i}$ is incorrect unless $k$ contains a square root of -1 . Instead we define them in a different way and prove $[9$, Theorem 3.17 p.32] in Proposition 13.4 and Proposition 13.6. This paper also contains

\footnotetext{
${ }^{3}$ The construction of the total power operations given here is also slightly different from the construction given in my unpublished notes on the operations written in 1996.
} 
all the results about cohomological operations necessary for the proof of the Milnor Conjecture given in [11].

The first draft of this paper was written in April 1996 i.e., exactly five years ago. During these years I discussed problems related to operations in motivic cohomology with a lot of people and I am greatful to all of them for these conversations. I would like to especially thank Mike Hopkins, Fabien Morel, Charles Rezk and Alexander Vishik.

This paper was written while I was a member of the Institute for Advanced Study in Princeton and, part of the time, an employee of the Clay Mathematics Institute. I am very grateful to both institutions for their support.

\section{Motivic cohomology and operations}

For any $p, q \in \mathbf{Z}$ and any abelian group $A$ the motivic cohomology of a smooth scheme $X$ are defined as hypercohomology

$$
H^{p, q}(X, A):=\mathbf{H}^{p}\left(X_{N i s}, A(q)\right)
$$

where $A(q)=\mathbf{Z}(q) \otimes A$ and $\mathbf{Z}(q)$ is a certain complex of sheaves of abelian groups on $(S m / k)_{N i s}$. Let $K(p, q, A)$ be the simplicial abelian sheaf corresponding to the complex $A(q)[p]$. Considered as a pointed simplicial sheaf of sets it defines an object of the pointed motivic homotopy category $H_{\bullet}(k)$. The simplicial sheaves $K(p, q, A)$ are $\mathbf{A}^{1}$-local and for a smooth scheme $X$ one has

$$
\operatorname{Hom}_{H \bullet(k)}\left(X_{+}, K(p, q, A)\right)=H^{p, q}(X, A)
$$

i.e. the objects $K(p, q, A)$ represent motivic cohomology on $H_{\text {• }}$ For any pointed simplicial sheaf $F$ • we define its reduced motivic cohomology by

$$
\tilde{H}^{p, q}\left(F_{\bullet}, A\right):=\operatorname{Hom}_{H \bullet(k)}\left(F_{\bullet}, K(p, q, A)\right)
$$

Let $R$ be a commutative ring with unit. Then $R(q)$ are complexes of sheaves of free $R$-modules and one has multiplication maps

$$
R(q) \otimes R\left(q^{\prime}\right) \rightarrow R\left(q+q^{\prime}\right)
$$

which define morphisms of pointed sheaves

$$
K(p, q, R) \wedge K\left(p^{\prime}, q^{\prime}, R\right) \rightarrow K\left(p+p^{\prime}, q+q^{\prime}, R\right)
$$


Recall that for a smooth scheme $X$ over $k$ we denote by $\mathbf{Z}_{t r}(X)$ the presheaf on $S m / k$ which takes $U$ to the group of cycles on $U \times X$ which are finite and equidimensional over $U$. For an abelian group $A$ we denote by $A_{t r}(X)$ the presheaf $\mathbf{Z}_{t r}(X) \otimes A$.

Let $K_{n, A}$ be the pointed sheaf of sets associated with the presheaf

$$
K_{n, A}^{\text {pre }}: U \mapsto A_{t r}\left(\mathbf{A}^{n}\right)(U) / A_{t r}\left(\mathbf{A}^{n}-\{0\}\right)(U)
$$

where the quotient is the quotient in the category of abelian groups. A section of $K_{n}^{\text {pre }}$ on $U$ is an equivalence class of cycles on $U \times \mathbf{A}^{n}$ with coefficients in $A$ which are equidimensional and finite over $U$. If $R$ is a commutative ring then we have the multiplication maps

$$
K_{n, R} \wedge K_{m, R} \rightarrow K_{n+m, R}
$$

which send the pair of cycles $Z, Z^{\prime}$ to the external product cycle $Z \otimes Z^{\prime}$. The following result is proved in [3].

Theorem 2.1 There are $\mathbf{A}^{1}$-weak equivalences $K_{n, R} \rightarrow K(2 n, n, R)$ which are compatible with the multiplication maps.

For pointed sheaves $F_{\bullet}, G_{\bullet}$ the morphisms (2.1) define multiplication maps

$$
H^{*, *}\left(F_{\bullet}, R\right) \otimes H^{*, *}\left(G_{\bullet}, R\right) \rightarrow H^{*, *}\left(F_{\bullet} \wedge G_{\bullet}, R\right)
$$

which we denote, on elements, by $w \otimes w^{\prime} \mapsto w \wedge w^{\prime}$. If $G_{\bullet}=F_{\text {. the com- }}$ position of (2.3) with the map defined by the diagonal $F_{\bullet} \rightarrow F_{\bullet} \wedge F_{\text {• defines }}$ multiplication maps

$$
H^{*, *}\left(F_{\bullet}, R\right) \otimes H^{*, *}\left(F_{\bullet}, R\right) \rightarrow H^{*, *}\left(F_{\bullet}, R\right)
$$

which we denote on elements by $w \otimes w^{\prime} \mapsto w w^{\prime}$.

Theorem 2.2 The morphisms (2.4) define, for any $F_{\bullet}$, a structure of an associative $R$-algebra on $\tilde{H}^{*, *}\left(F_{\bullet}, R\right)$ which is graded commutative with respect to the first grading.

Proof: Standard arguments from homological algebra together with the fact that complexes $R(q)$ do not have cohomology in dimensions $>2 q$ imply that it is sufficient to check that the multiplication maps

$$
K(2 q, q, R) \wedge K\left(2 q^{\prime}, q^{\prime}, R\right) \rightarrow K\left(2\left(q+q^{\prime}\right), q+q^{\prime}, R\right)
$$


are associative and commutative in $H_{\bullet}$. By Theorem 2.1 it is sufficient to check that the multiplication maps (2.2) are commutative and associative in $H_{\text {. }}$ The associativity condition clearly holds on the level of sheaves. To prove commutativity we should show that permutation of coordinates on $\mathbf{A}^{n}$ acts trivially on $K_{n, R}$. The action of the permutation group on $K_{n, R}$ extends to an action of $G L_{n}$. A transposition is $\mathbf{A}^{1}$-homotopic to the automorphism given by $\left(x_{1}, \ldots, x_{n}\right) \mapsto\left(-x_{1}, \ldots, x_{n}\right)$. It is therefore sufficient to check that this automorphism is the identity in $H_{\bullet}$. Consider for simplicity of notations the case of one variable i.e. the automorphism $\phi$ of $\mathbf{Z}_{t r}\left(\mathbf{A}^{1}\right) / \mathbf{Z}_{t r}\left(\mathbf{A}^{1}-\{0\}\right)$ defined by $x \mapsto-x$. The sheaf $\mathbf{Z}_{t r}\left(\mathbf{A}^{1}\right) / \mathbf{Z}_{t r}\left(\mathbf{A}^{1}-\{0\}\right)$ is isomorphic to the sheaf $\mathbf{Z}_{t r}\left(\mathbf{P}^{1}\right) / \mathbf{Z}_{t r}\left(\mathbf{P}^{1}-\{0\}\right)$ which is weakly equivalent to $\mathbf{Z}_{t r}\left(\mathbf{P}^{1}\right) / \mathbf{Z}$ where the embedding $\mathbf{Z} \rightarrow \mathbf{P}^{1}$ corresponds to the point $\infty$. Under this weak equivalence our automorphism becomes the automorphism of $\mathbf{Z}_{t r}\left(\mathbf{P}^{1}\right) / \mathbf{Z}$ defined by $z \mapsto$ $-z$. Denote this automorphism by $\psi$. One can easily see now that to prove that $\phi$ is identity in $H_{\bullet}$ it is sufficient to construct a section $h$ of $\mathbf{Z}_{t r}\left(\mathbf{P}^{1}\right)$ on $\mathbf{P}^{1} \times \mathbf{A}^{1}$ such that

$$
h_{\mathbf{P}^{1} \times\{1\}}-h_{\mathbf{P}^{1} \times\{1\}}=I d-\psi
$$

Let $\left(\left(z_{0}: z_{1}\right), t\right)$ be the coordinates on $\mathbf{P}^{1} \times \mathbf{A}^{1}$ and $\left(x_{0}: x_{1}\right)$ the coordinates on $\mathbf{P}^{1}$. Then the cycle of the closed subscheme in $\mathbf{P}^{1} \times \mathbf{A}^{1} \times \mathbf{P}^{1}$ given by the equation $z_{0} x_{1}^{2}+t z_{1} x_{0} x_{1}+\left(t^{2}-1\right) x_{0}^{2}$ defines a section of $\mathbf{Z}_{t r}\left(\mathbf{P}^{1}\right)$ on $\mathbf{P}^{1} \times \mathbf{A}^{1}$ which satisfies (2.5).

Denote by $H^{*, *}$ the ring

$$
H^{*, *}(\operatorname{Spec}(k), R)=\tilde{H}^{*, *}\left(S^{0}, R\right)
$$

Then for any $F$. the multiplication maps (2.3) define a structure of $H^{*, *}$ module on $\tilde{H}^{*, *}\left(F_{\bullet}, R\right)$. Theorem 2.2 immediately implies the following fact.

Corollary 2.3 The multiplication map (2.4) factors through an $H^{*, *}$-module map

$$
H^{*, *}\left(F_{\bullet}, R\right) \otimes_{H^{*, *}} H^{*, *}\left(F_{\bullet}, R\right) \rightarrow H^{*, *}\left(F_{\bullet}, R\right)
$$

Let $S_{s}^{1}$ and $S_{t}^{1}$ be the circles in $H_{\bullet}$. We have canonical classes

$$
\begin{gathered}
\sigma_{s} \in \tilde{H}^{1,0}\left(S_{s}^{1}, R\right) \\
\sigma_{t} \in \tilde{H}^{1,1}\left(S_{t}^{1}, R\right)
\end{gathered}
$$


Multiplication with these classes gives us suspension morphisms

$$
\begin{gathered}
\tilde{H}^{p, q}\left(F_{\bullet}, R\right) \rightarrow \tilde{H}^{p+1, q}\left(F_{\bullet} \wedge S_{s}^{1}, R\right) \\
\tilde{H}^{p, q}\left(F_{\bullet}, R\right) \rightarrow \tilde{H}^{p+1, q+1}\left(F_{\bullet} \wedge S_{t}^{1}, R\right)
\end{gathered}
$$

Theorem 2.4 The suspension morphisms are isomorphisms.

Proof: Let $\tilde{\mathbf{Z}}$ be the functor from sheaves of pointed sets to sheaves of abelian groups which sends a sheaf of sets to the freely generated sheaf of abelian groups with the distinguished point set to be zero. Let further $N$ be the normalized chain complex functor from simplicial abelian sheaves to the complexes of abelian sheaves. Then for any $F_{\bullet}$ one has

$$
\tilde{H}^{p, q}\left(F_{\bullet}, A\right)=H_{o m}\left(N \tilde{\mathbf{Z}}\left(F_{\bullet}\right), A(q)[p]\right)
$$

where $D$ is the derived category of complexes of abelian sheaves in the Nisnevich topology. The fact that (2.7) is an isomorphism follows from the fact that $N \mathbf{Z}$ takes smash product to tensor product (modulo a quasiisomorphism) and that $N \mathbf{Z}\left(S_{s}^{1}\right)$ is quasi-isomorphic to $\mathbf{Z}[1]$.

Consider the suspension morphism

$$
(-) \wedge \sigma_{T}: \tilde{H}^{p, q}\left(F_{\bullet}, A\right) \rightarrow \tilde{H}^{p+2, q+1}\left(F_{\bullet} \wedge T, A\right)
$$

given by multiplication with the class $\sigma_{T} \in \tilde{H}^{2,1}(T)$. Since (2.7) is an isomorphism and we have an $\mathbf{A}^{1}$-weak equivalence $S_{s}^{1} \wedge S_{t}^{1} \rightarrow T=h_{\mathbf{A}^{1}} / h_{\left(\mathbf{A}^{1}-\{0\}\right)}$, to show that (2.8) is an isomorphism it is sufficient to show that $(-) \wedge \sigma_{T}$ is an isomorphism. A standard argument allows one to reduce the problem to the case when $F_{\mathbf{~}}=\left(h_{U}\right)_{+}$for a smooth scheme $U$ over $S$. Open excision implies that $T=\mathbf{P}^{1} / \mathbf{A}^{1}$ and we get a split short exact sequence

$$
0 \rightarrow \tilde{H}^{*, *}\left(\left(h_{U}\right)_{+} \wedge T, A\right) \rightarrow H *, *\left(U \times \mathbf{P}^{1}, A\right) \rightarrow H^{*, *}\left(U \times \mathbf{A}^{1}, A\right) \rightarrow 0
$$

Consider the morphism of sequences:

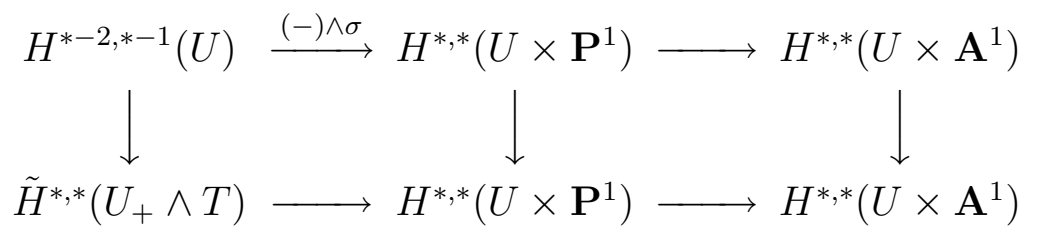

where $\sigma$ is the restriction of $\sigma_{T}$ to $\mathbf{P}^{1}$, the first vertical arrow is $(-) \wedge \sigma_{T}$ and the rest of vertical arrows are identities. The fact that $(-) \wedge \sigma_{T}$ is an isomorphism follows now from Lemma 2.5 below. 
Lemma 2.5 The upper sequence in (2.9) is a short exact sequence.

Proof: By [10, Cor. 2] we have natural isomorphisms

$$
H^{p, q}(U, \mathbf{Z}) \rightarrow C H^{q}(U, 2 q-p)
$$

where the target are Bloch's higher Chow groups and the proof immediately shows that we have similar isomorphisms for all groups of coefficients

$$
H^{p, q}(U, A) \rightarrow C H^{q}(U, 2 q-p, A)
$$

Consider the diagram:

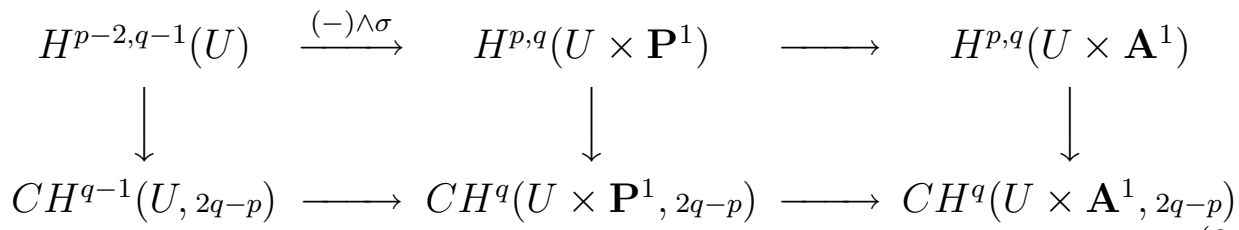

where in the lower line the first morphism is given by covariant functoriality for the closed embedding $U \rightarrow U \times \mathbf{P}^{1}$ at the infinity and the second morphism is given by the contravariant functoriality for the open embedding $U \times \mathbf{A}^{1} \rightarrow U \times \mathbf{P}^{1}$. One verifies using the explicit form of the isomorphism (2.10) that both squares in (2.11) commute. We conclude that the upper line is a short exact sequence since the lower one is a short exact sequence by Bloch's Localization Theorem [1].

We define a bistable cohomological operation of bidegree $(i, j)$ in motivic cohomology with coefficients in $R$ as a collection of natural transformations of functors on $H_{\bullet}$

$$
\phi_{p, q}: \tilde{H}^{p, q}(-, R) \rightarrow \tilde{H}^{p+i, q+j}(-, R)
$$

which commutes with the suspension morphisms i.e. such that for $x \in \tilde{H}^{p, q}$ one has

$$
\begin{aligned}
& \phi_{p+1, q}\left(x \wedge \sigma_{s}\right)=\phi_{p, q}(x) \wedge \sigma_{s} \\
& \phi_{p+1, q+1}\left(x \wedge \sigma_{t}\right)=\phi_{p, q}(x) \wedge \sigma_{t}
\end{aligned}
$$

Denote by $\sigma_{T}$ the canonical element in $\tilde{H}^{2,1}(T, R)$ where $T=\mathbf{A}^{1} / \mathbf{A}^{1}-\{0\}$. 
Proposition 2.6 There is a bijection between the set of bistable cohomological operation of bidegree $(i, j)$ and the collections of natural transformations

$$
\phi_{n}: \tilde{H}^{2 n, n}(-, R) \rightarrow \tilde{H}^{2 n+i, n+j}(-, R)
$$

given for all $n \geq 0$ such that

$$
\phi_{n+1}\left(x \wedge \sigma_{T}\right)=\phi_{n}(X) \wedge \sigma_{T}
$$

Proof: Since $T=S_{s}^{1} \wedge S_{t}^{1}$ and $\sigma_{T}=\sigma_{s} \wedge \sigma_{t}$, the restriction of a bistable operation to groups of degree $(2 n, n)$ satisfies $(2.13)$. On the other hand, for a family $\phi_{n}$ we can construct $\phi_{p, q}$ as follows. For $F$ • we have

$$
\tilde{H}^{p, q}\left(F_{\bullet}\right)=\tilde{H}^{p+a+b, q+b}\left(S_{t}^{a} \wedge S_{s}^{b} \wedge F_{\bullet}\right)
$$

taking $a=2 q-p+b$ and taking $b$ to be greater or equal to $\max \{0,-q, p-2 q\}$ we get:

$$
\tilde{H}^{p, q}\left(F_{\bullet}\right)=\tilde{H}^{2(q+b), q+b}\left(S_{t}^{a} \wedge S_{s}^{b} \wedge F_{\bullet}\right)
$$

where $a, b, q+b \geq 0$. Using these isomorphisms, the operation $\phi_{q+b}$ defines a map

$$
\phi_{p, q}: \tilde{H}^{p, q}\left(F_{\bullet}\right) \rightarrow \tilde{H}^{p+i, q+j}\left(F_{\bullet}\right)
$$

The condition (2.13) implies that this map does not depend on the choice of $b$ and that the maps $\phi_{p, q}$ all $(p, q)$ satisfy $(2.12)$.

Combining Theorem 2.1 and Proposition 2.6 we get.

Proposition 2.7 There is a bijection between the set of bistable cohomological operations of bidegree $(i, j)$ and collections of motivic cohomology classes $\phi_{n} \in \tilde{H}^{2 n+i, n+j}\left(K_{n, R}, R\right)$ such that the restriction of $\phi_{n+1}$ to $K_{n, R} \wedge T$ is $\phi_{n} \wedge \sigma_{T}$.

Let $c: S_{s}^{1} \rightarrow S_{s}^{1} \vee S_{s}^{1}$ be the map in $H_{\bullet}$ corresponding to the usual codiagonal on the (simplicial) circle.

Proposition 2.8 Let $F_{\bullet}$ be a pointed simplicial sheaf. Then for any $p, q$ the map

$$
\tilde{H}^{p, q}\left(S_{s}^{1} \wedge F_{\bullet}\right) \oplus \tilde{H}^{p, q}\left(S_{s}^{1} \wedge F_{\bullet}\right) \rightarrow \tilde{H}^{p, q}\left(S_{s}^{1} \wedge F_{\bullet}\right)
$$

defined by the codiagonal $c$ is of the form $(a, b) \mapsto a+b$. 
Proof: It follows by adjunction argument from the fact that the map of the free abelian groups defined by $c$ is isomorphic, in the derived category of sheaves of abelian groups, to the diagonal map $\mathbf{Z}[1] \rightarrow \mathbf{Z}[1] \oplus \mathbf{Z}[1]$.

Corollary 2.9 Let $\alpha: \tilde{H}^{p, q} \rightarrow \tilde{H}^{r, s}$ be a cohomological operation. Then for any pointed simplicial sheaf $F$. the map:

$$
\tilde{H}^{p, q}\left(S_{s}^{1} \wedge F_{\bullet}\right) \rightarrow \tilde{H}^{r, s}\left(S_{s}^{1} \wedge F_{\bullet}\right)
$$

defined by $\alpha$ is a homomorphism of abelian groups.

Proof: Follows from Proposition 2.8 using the naturality of $\alpha$ with respect to the map defined by the codiagonal $c$.

Corollary 2.10 Let $\alpha: \tilde{H}^{*, *} \rightarrow \tilde{H}^{*+i, *+j}$ be a bistable cohomological operation. Then for any $F$. the map

$$
\tilde{H}^{*, *}\left(F_{\bullet}\right) \rightarrow \tilde{H}^{*+i, *+j}\left(F_{\bullet}\right)
$$

defined by $\alpha$ is a homomorphism of abelian groups.

Proof: Follows from Corollary 2.9.

\section{Operations $\tilde{H}^{2 d, d} \rightarrow \tilde{H}^{2 d+*, d+i}$ for $i \leq 0$}

In this section it will be convenient for us to use a different model for the space $K_{n, A}$. We define $K_{n, A}^{\prime}$ as the sheaf which sends $U$ to the group of cycles with coefficients in $R$ on $U \times \mathbf{A}^{n}$ which are equidimensional and of relative dimension zero over $U$. The following theorem is proved in [10].

Theorem 3.1 There is an isomorphism $K_{n, A} \rightarrow K_{n, A}^{\prime}$ in the $\mathbf{A}^{1}$-homotopy category of sheaves with transfers.

As a corollary we get the following result.

Corollary 3.2 The pointed sheaves $K_{n, A}$ and $K_{n, A}^{\prime}$ are isomorphic in $H_{\bullet}$.

Given a pointed sheaf $F$ define its standard simplicial resolution as the simplicial sheaf $G_{\bullet} F$ with terms of the form

$$
G_{i} F=\left(\coprod_{X_{0} \rightarrow \ldots \rightarrow X_{i} ; f \in F\left(X_{i}\right)-\{*\}} X_{0}\right)_{+}
$$


where the coproduct is taken over all sequences of morphisms of length $i$ in some small subcategory equivalent to $S m / k$ (see [3] for more details). One verifies easily that the obvious morphism $G_{\bullet} F \rightarrow F$ is a weak equivalence of pointed simplicial presheaves.

For a cycle $Z$ on $X$ denote by $\operatorname{Supp}(Z)$ the closure of the set of points which appear in $Z$ with nonzero multiplicity. Consider $G_{\bullet} K_{n, A}^{\prime} \times \mathbf{A}^{n}$. For each $i$ let $F_{n, i}$ be the open subset in

$$
G_{i} K_{n, A}^{\prime} \times \mathbf{A}^{n}=\left(\coprod_{X_{0} \rightarrow \ldots \rightarrow X_{i} ; Z \in z\left(X_{i} \times \mathbf{A}^{n} / X_{i}\right)-\{0\}} X_{0} \times \mathbf{A}^{n}\right)_{+}
$$

whose component corresponding to $\left(X_{0} \rightarrow \ldots \rightarrow X_{i} ; Z\right)$ is the complement to $X_{0} \times_{X_{i}} \operatorname{Supp}(Z)$. The following lemma is straightforward.

Lemma 3.3 The collection of subsheaves $F_{n, i}$ forms a simplicial subsheaf in $G_{\bullet} K_{n, A}^{\prime} \times \mathbf{A}^{n}$.

Proposition 3.4 The composition $F_{n, \bullet} \rightarrow G_{\bullet} K_{n, A}^{\prime} \times \mathbf{A}^{n} \rightarrow K_{n, A}^{\prime}$ is $\mathbf{A}^{1}$ homotopic to the zero morphism.

Proof: To prove the proposition it is sufficient to construct for any $X$ and any $Z$ in $\operatorname{Hom}\left(X, K_{n, A}^{\prime}\right)=z\left(X \times \mathbf{A}^{n} / X\right)$ an $\mathbf{A}^{1}$-homotopy from the map

$$
X \times \mathbf{A}^{n}-\operatorname{Supp}(Z) \rightarrow X \rightarrow K_{n, A}^{\prime}
$$

such that these homotopies are natural in $X$. Consider the map

$$
h:\left(X \times \mathbf{A}^{n}-\operatorname{Supp}(Z)\right) \times \mathbf{A}^{n} \times \mathbf{A}^{1} \rightarrow\left(X \times \mathbf{A}^{n}-S u p p(Z)\right) \times \mathbf{A}^{n}
$$

which sends $(x, u, v, t)$ to $(x, u, u(1-t)+v t$. This map is flat over the complement to $X \times \Delta\left(\mathbf{A}^{n}\right)$ in the target. Consider the pull-back $p^{*}(Z)$ of $Z$ along the map $p: X \times \mathbf{A}^{n}-\operatorname{Supp}(Z) \rightarrow X$. It is a cycle on the target of $h$ and the support of this cycle does not intersect $X \times \Delta\left(\mathbf{A}^{n}\right)$. Therefore, the flat pull-back $h^{*}\left(p^{*}(Z)\right)$ is defined. One verifies easily that this cycle is equidimensional over $\left(X \times \mathbf{A}^{n}-\operatorname{Supp}(Z)\right) \times \mathbf{A}^{1}$ of relative dimension zero and hence defines a map:

$$
H:\left(X \times \mathbf{A}^{n}-\operatorname{Supp}(Z)\right) \times \mathbf{A}^{1} \rightarrow K_{n, A}^{\prime}
$$

The restriction of $h^{*}\left(p^{*}(Z)\right)$ to $t=0$ is $p^{*}(Z)$ and the restriction to $t=1$ is the zero cycle. Therefore, $H$ is a homotopy of the required form. It is clear that our construction is functorial in $X$. 
Corollary 3.5 The object $K_{n, A}^{\prime}$ is a retract, in $H_{\bullet}$, of $G_{\bullet} K_{n, A}^{\prime} \times \mathbf{A}^{n} / F_{n, \bullet}$.

We will often use below the following result.

Lemma 3.6 Let $k$ be a field, $X$ be smooth scheme over $k$ and $Z$ a closed subscheme in $X$ everywhere of codimension at least $c$. Then $\tilde{H}^{*, q}(X /(X-$ $Z))=0$ for $q<c$ and

$$
\tilde{H}^{p, c}(X /(X-Z), A)=\left\{\begin{array}{cl}
\oplus_{z \in Z^{c}} A & \text { for } p=2 c \\
0 & \text { for } p \neq 2 c
\end{array}\right.
$$

where $Z^{c}$ is the set of points of $Z$ which are of codimension $c$ in $X$.

Proposition 3.7 For any $n \geq 0, m<n$ and any abelian groups $A, B$ one has

$$
\tilde{H}^{*, m}\left(K_{n, A}, B\right)=0
$$

Proof: Follows immediately from Corollary 3.5 and Lemma 3.6.

Proposition 3.8 For any $n>0$ and any abelian groups $A, B$ one has

$$
\tilde{H}^{p, n}\left(K_{n, A}, B\right)=\left\{\begin{array}{cl}
\operatorname{Hom}(A, B) & \text { for } p=2 n \\
0 & \text { for } p<2 n
\end{array}\right.
$$

Proof: The fact that $\tilde{H}^{p, n}\left(K_{n, A}, B\right)=0$ for $p<2 n$ follows immediately from Corollary 3.5 and Lemma 3.6. Consider the case $p=2 n$. We have an obvious map from $\operatorname{Hom}(A, B)$ to $\tilde{H}^{2 n, n}\left(K_{n, A}, B\right)$. On the other hand, an element of $\tilde{H}^{2 n, n}\left(K_{n, A}, B\right)$ considered as an operation defines a map

$$
A=\tilde{H}^{2 n, n}\left(T^{n}, A\right) \rightarrow \tilde{H}^{2 n, n}\left(T^{n}, B\right)=B
$$

Since $T=S_{s}^{1} \wedge S_{t}^{1}$ and $n>0$, Corollary 2.9 implies that this map is a homomorphism. Therefore, it is sufficient to show that an element $\alpha$ of $\tilde{H}^{2 n, n}\left(K_{n, A}, B\right)$ which acts trivially on $\tilde{H}^{2 n, n}\left(T^{n}, A\right)$ is zero. By Corollary 3.5 any $\alpha$ is determined by its action on objects of the form $X \times \mathbf{A}^{n} /\left(X \times \mathbf{A}^{n}-Z\right)$ where $Z$ is a closed subset equidimensional of relative dimension 0 over $\mathbf{A}^{n}$. Let $Z_{\text {sing }}$ be the closed subset of singular points of $Z$. Since $k$ is perfect, $Z_{\text {sing }}$ is of codimension at least $n+1$ in $X \times \mathbf{A}^{n}$. Lemma 3.6 implies that the motivic cohomology of weight $n$ of $X \times \mathbf{A}^{n} /\left(X \times \mathbf{A}^{n}-Z\right)$ map to the motivic 
cohomology of weight $n$ of $\left(X \times \mathbf{A}^{n}-Z_{\text {sing }}\right) /\left(X \times \mathbf{A}^{n}-Z\right)$ isomorphically. It remains to show that $\alpha$ acts trivially on

$$
\tilde{H}^{2 n, n}\left(\left(X \times \mathbf{A}^{n}-Z_{\text {sing }}\right) /\left(X \times \mathbf{A}^{n}-Z\right), A\right)
$$

The normal bundle to $Z-Z_{\text {sing }}$ in $X \times \mathbf{A}^{n}-Z_{\text {sing }}$ is trivial. Hence, by the homotopy purity theorem [6], we have a weak equivalence

$$
\left(X \times \mathbf{A}^{n}-Z_{\text {sing }}\right) /\left(X \times \mathbf{A}^{n}-Z\right)=\Sigma_{T}^{n}\left(\left(Z-Z_{\text {sing }}\right)_{+}\right)
$$

let $Z_{i}, i=1, \ldots, m$ be the connected components of $Z-Z_{\text {sing }}$. Then we have a map

$$
\Sigma_{T}^{n}\left(\left(Z-Z_{\text {sing }}\right)_{+}\right) \rightarrow \vee_{i=1}^{m} T^{n}
$$

and $H^{j, 0}\left(Z_{i}\right)$ is non-zero only for $j=0$ where it is $A$, this map defines an isomorphism on $\tilde{H}^{2 n, n}(-, A)$. We conclude that $\alpha$ acts by zero since by assumption it acts by zero on $\tilde{H}^{2 n, n}\left(T^{n}, A\right)$.

\section{Thom isomorphism and Euler classes}

If $E$ is a vector bundle and $\mathbf{P}(E)$ is the projective bundle defined by $E$ then the line bundle $\mathcal{O}(-1)$ on $\mathbf{P}(E)$ gives a class in $H^{2,1}(\mathbf{P}(E), \mathbf{Z})$ which we denote by $\sigma$. The following result is proved in [][].

Theorem 4.1 For any smooth $X$ over $k$ and a vector bundle $E$ on $X$ of dimension $d$, the elements $1, \sigma, \ldots, \sigma^{d-1}$ form a basis of the $H^{*, *}$-module $H^{*, *}(\mathbf{P}(E), \mathbf{Z})$.

The key ingredient of the proof of this theorem is the following lemma which we will also use directly.

Lemma 4.2 Let 0 be the image of the point $(0, \ldots, 0)$ under the standard embedding $\mathbf{A}^{n} \rightarrow \mathbf{P}^{n}$. Let further $f: \mathbf{P}^{n} \rightarrow T^{n}$ be the composition

$$
\mathbf{P}^{n} \rightarrow \mathbf{P}^{n} /\left(\mathbf{P}^{n}-0\right) \cong \mathbf{A}^{n} /\left(\mathbf{A}^{n}-0\right)=T^{n}
$$

and $t$ the tautological class in $\tilde{H}^{2 n, n}\left(T^{n}, \mathbf{Z}\right)$. Then $f^{*}(t)=(-\sigma)^{n}$.

Recall that for a vector bundle $E$ on $X$ we denote by $T h(E)$ the pointed sheaf $E /(E-z(X))$ where $z: X \rightarrow E$ is the zero section. Consider the projective bundle $\mathbf{P}(E \oplus \mathcal{O})$. We have two morphisms

$$
X=\mathbf{P}(\mathcal{O}) \rightarrow \mathbf{P}(E \oplus \mathcal{O})
$$




$$
\mathbf{P}(E) \rightarrow \mathbf{P}(E \oplus \mathcal{O})
$$

The complement to the image of the second morphism is $E$ and open excision implies that

$$
T h(E)=\mathbf{P}(E \oplus \mathcal{O}) / \mathbf{P}(E \oplus \mathcal{O})-X
$$

On the other hand the map $\mathbf{P}(E) \rightarrow \mathbf{P}(E \oplus \mathcal{O})-X$ is locally of the form $\mathbf{P}^{n-1} \rightarrow \mathbf{P}^{n}-p t$ and therefore it is an $\mathbf{A}^{1}$-weak equivalence. We conclude that the morphism

$$
\mathbf{P}(E \oplus \mathcal{O}) / \mathbf{P}(E) \rightarrow T h(E)
$$

is a weak equivalence. If $f: E \rightarrow E^{\prime}$ is a monomorphism of vector bundles and $\mathbf{P}(f): \mathbf{P}(E) \rightarrow \mathbf{P}\left(E^{\prime}\right)$ is the corresponding morphism of projective bundles then $\mathbf{P}(f)^{*}(\mathcal{O}(-1))=\mathcal{O}(-1)$. Together with Theorem 4.1 this implies that the map on motivic cohomology defined by $\mathbf{P}(E) \rightarrow \mathbf{P}(E \oplus \mathcal{O})$ is a split mono and that there is a unique class in $\tilde{H}^{2 d, d}(T h(E), \mathbf{Z})$ whose image in the cohomology of $\mathbf{P}(E \oplus \mathcal{O})$ is of the form $(-\sigma)^{d}+\sum_{i<d} a_{i} \sigma^{i}$. This class is called the Thom class of $E$ and denoted $t_{E}$.

The obvious "diagonal" map $d: T h(E) \rightarrow X_{+} \wedge T h(E)$ defines multiplication $(x, y) \mapsto d^{*}(x \wedge y)$ with $x \in H^{*, *}(X), y \in \tilde{H}^{*, *}(T h(E))$ and $d^{*}(x \wedge y) \in \tilde{H}^{* * *}(T h(E))$. By abuse of notation we will write $x y$ instead of $d^{*}(x \wedge y)$.

Proposition 4.3 For any pointed simplicial sheaf $F_{\bullet}$ the map $a \mapsto a t_{E}$ from $\tilde{H}^{*, *}\left(F_{\bullet} \wedge X_{+}\right)$to $\tilde{H}^{*+2 d, *+d}\left(F_{\bullet} \wedge T h(E)\right)$ is an isomorphism.

Proof: A standard argument shows that it is sufficient to prove the proposition for $F_{\bullet}=p t$. In this case it follows immediately from out definition of the Thom class and the projective bundle theorem.

Corollary 4.4 The Thom class $t_{E}$ is a unique class in $\tilde{H}^{2 d, d}(T h(E))$ whose restriction to any generic point of $X$ is the tautological class in $\tilde{H}^{2 d, d}\left(T^{d}\right)$.

Proof: The fact that the restriction of $t_{E}$ to the generic point is the tautological class follows from Lemma 4.2. The fact that $t_{E}$ is determined by this condition follows from Proposition 4.3 and the fact that if $j: \amalg \operatorname{Spec}\left(K_{i}\right) \rightarrow X$ is the embedding of the generic points of $X$, then $j^{*}$ defines an isomorphism on $H^{0,0}$.

For a vector bundle $E$ define the Euler class $e(E)$ in $H^{2 d, d}(X)$ as the restriction of $t_{E}$ with respect to the zero section map $X_{+} \rightarrow T h(E)$. 
Lemma 4.5 Let $L$ be a line bundle. Then $e(L)$ coincides with the canonical class of $L$ in $H^{2,1}$. In particular, for two line bundles $L, L^{\prime}$ one has e $(L \otimes$ $\left.L^{\prime}\right)=e(L)+e\left(L^{\prime}\right)$.

Proof: Let $\sigma+c$ be the image of $t_{L}$ in $H^{2,1}(\mathbf{P}(L \oplus \mathcal{O}))$. The restriction of $\sigma$ to $\mathbf{P}(\mathcal{O})$ is $\mathcal{O}(-1)$ for $\mathcal{O}$ i.e. zero. Therefore, $e(L)=c$. On the other hand $c$ is defined by the condition that the restriction of $\sigma+c$ to $\mathbf{P}(L)$ is zero. Since the restriction of $\sigma$ is the class of $-L$ we conclude that $e(L)=L$.

Lemma 4.6 Let $E, E^{\prime}$ be two vector bundles. Then $e\left(E \oplus E^{\prime}\right)=e(E) e\left(E^{\prime}\right)$.

Proof: Corollary 4.4 implies that the Thom class for the sum of two bundles is the smash product of Thom classes. In particular, $e\left(E \oplus E^{\prime}\right)=e(E) e\left(E^{\prime}\right)$.

Lemma 4.7 Let $f: E \rightarrow E^{\prime}$ be a monomorphism of vector bundles on a quasi-projective scheme $X$ such that $E^{\prime} / E$ is again a vector bundle and $\operatorname{th}(f): T h(E) \rightarrow T h\left(E^{\prime}\right)$ be the corresponding map of Thom spaces. Then $\operatorname{th}(f)^{*}\left(t_{E}^{\prime}\right)=t_{E} e\left(E / E^{\prime}\right)$.

Proof: Since $X$ is quasi-projective we can find an affine torsor $X^{\prime} \rightarrow X$ such that $X^{\prime}$ is affine. Since motivic cohomology of $X$ and $X^{\prime}$ are the same it is sufficients to prove the lemma for an affine $X$. Over an affine $X$ the sequence $E \rightarrow E^{\prime} \rightarrow E^{\prime} / E$ splits and we have $E^{\prime}=E \oplus E^{\prime} / E$. Let $d$ : $T h\left(E \oplus E^{\prime} / E\right) \rightarrow T h(E) \wedge T h\left(E^{\prime} / E\right)$ be the obvious morphism. Corollary 4.4 implies that $t_{E^{\prime}}=d^{*}\left(t_{E} \wedge t_{E^{\prime} / E}\right)$ which in turn implies the statement of the lemma.

\section{Total power operations}

Construction 5.1 Let $E, L$ be vector bundles on $X$ and $\phi: E \oplus L \rightarrow \mathcal{O}^{n}$ an isomorphism. For an cycle $Z$ on $E$ with coefficients in a commutative ring $R$ which is equidimensional and finite over $X$ consider the cycle on $L \times_{X} E \times_{X} L$ whose fiber over a point $(x, l)$ of $L$ is $Z_{x} \times l$. One verifies easily that this is a cycle equidimensional and finite over $L$. Identifying $E \times_{X} L$ with $\mathbf{A}_{X}^{n}$ by means of $\phi$ we get a section of $\mathbf{Z}_{t r}\left(\mathbf{A}^{N}\right)$ on $L$. The restriction of this section to $L-z(X)$ where $z: X \rightarrow L$ is the zero section lies in $\mathbf{Z}_{t r}\left(\mathbf{A}^{N}-\{0\}\right)$. Therefore, it gives a map of pointed sheaves $T h(L) \rightarrow K_{n, R}$ which we denote $a(Z)$. 
Lemma 5.2 The motivic cohomology class $\tilde{a}(Z)$ in $H^{2 \operatorname{dim}(E), \operatorname{dim}(E)}(X)$ defined by $a(Z)$ through the Thom isomorphism does not depend on the choice of $L$ and $\phi$.

Proof: Let $x$ be a generic point of $X, L_{x}$ the fiber of $L$ over $x$ and $\sum z_{i}$ the fiber of $Z$ over $x$. Then, for a generic point $l$ of $L_{x}$ the fiber of $\tilde{a}(Z)$ over $(x, l)$ is $\sum \phi\left(z_{i}, l\right)$. Given another pair $\left(L^{\prime}, \phi^{\prime}\right)$ consider the isomorphism

$$
L \oplus \mathcal{O}^{N^{\prime}} \rightarrow L \oplus E \oplus L^{\prime} \rightarrow L^{\prime} \oplus E \oplus L \rightarrow L^{\prime} \oplus \mathcal{O}^{N}
$$

and let $\psi: T h\left(L \oplus \mathcal{O}^{N^{\prime}}\right) \rightarrow T h\left(L \oplus \mathcal{O}^{N}\right)$ be the corresponding isomorphism of Thom spaces. We claim that

$$
\psi^{*}\left(a(Z) t_{N}^{\prime}\right)=a^{\prime}(Z) t_{N}
$$

on the level of actual cycles. Indeed, the fiber of $a(Z) t_{N^{\prime}}$ over a generic point $(x, l, u)$ of $L \oplus \mathcal{O}^{N^{\prime}}$ is $\sum\left(\phi\left(z_{i}, l\right), u\right)$ (in $\mathcal{O}^{N} \oplus \mathcal{O}^{N^{\prime}}$ ) which coincides with the fiber of $a^{\prime}(Z) t_{N}$ over $\psi(x, l, u)$. Using (5.1) and applying Lemma 4.7 to $\psi^{*}$ we get the statement of our lemma.

Construction 5.3 Let $G$ be a finite group, $r: G \rightarrow S_{n}$ a permutational representation of $G, U$ a smooth scheme with free action of $G$ and $L$ a vector bundle on $U / G$ given together with an isomorphism $\xi_{n} \oplus L \rightarrow \mathcal{O}^{N}$ where $\xi_{n}$ is the vector bundle of dimension $n$ on $U / G$ corresponding to $r$. Given any such collection and a cycle $Z$ on $X \times \mathbf{A}^{i}$ equidimensional and finite over $X$ define a map

$$
\tilde{P}(Z): X \wedge T h_{U / G}\left(L^{i}\right) \rightarrow K_{i N, R}
$$

as follows. Let $Z^{\otimes n}$ be the external power of $Z$. It is a cycle on $\left(X \times \mathbf{A}^{i}\right)^{n}$. Let $p^{*}\left(Z^{\otimes n}\right)$ be its flat pull-back to $\left(X \times \mathbf{A}^{i}\right)^{n} \times U$. Since $p^{*}\left(Z^{\otimes n}\right)$ is invariant under the action of $G$ and the action of $G$ on $U$ is free there exists a unique cycle $Z^{\prime}$ on $\left(\left(X \times \mathbf{A}^{i}\right)^{n} \times U\right) / G$ whose pull-back to $\left(X \times \mathbf{A}^{i}\right)^{n} \times U$ is $Z$. One verifies easily that $Z^{\prime}$ is finite and equidimensional over $\left(X^{n} \times U\right) / G$. Therefore, we can pull it back to a cycle $Z^{\prime \prime}$ on $X \times\left(\mathbf{A}^{i n} \times U\right) / G$ by means of the diagonal map $X \rightarrow X^{i}$. The scheme $\left(\mathbf{A}^{i n} \times U\right) / G$ is the vector bundle $\xi_{n}^{i}$ over $U / G$ and we define $\tilde{P}(Z)$ as $a\left(Z^{\prime \prime}\right)$. One verifies immediately that if $Z$ lies in $X \times\left(\mathbf{A}^{i}-\{0\}\right)$ then $\tilde{P}(Z)=0$.

This construction defines a morphism of pointed sheaves:

$$
\tilde{P}: K_{i, R} \wedge T h_{U / G}\left(L^{i}\right) \rightarrow K_{i N, R}
$$


Since smash products preserve $\mathbf{A}^{1}$-weak equivalences, this morphism defines an operation

$$
\tilde{P}: \tilde{H}^{2 i, i}(-, R) \rightarrow \tilde{H}^{2 i N, i N}\left(-\wedge T h_{U / G}\left(L^{i}\right), R\right)
$$

By Lemma 4.3 the Thom isomorphism defines a morphism in $H_{\bullet}$ of the form

$$
P: K_{i, R} \wedge(U / G)_{+} \rightarrow K_{i n, R}
$$

or, equivalently an operation

$$
P: \tilde{H}^{2 i, i}(-, R) \rightarrow \tilde{H}^{2 i n, i n}\left(-\wedge(U / G)_{+}, R\right)
$$

such that

$$
\tilde{P}(x)=P(x) t_{L^{i}}
$$

Lemma 5.2 implies immediately the following result.

Lemma 5.4 The operation $P=P_{G, r, U, L, \phi}$ does not depend on the choice of $L$ and $\phi$.

If $U$ is a quasi-projective scheme with a free action of $G$ then we can find, using the standard trick, an affine smooth scheme $\tilde{U}$ with a free action of $G$ and an equivariant morphism $\tilde{U} \rightarrow U$. Since any vector bundle $E$ on an affine scheme is "invertible", i.e. there is an $L$ such that $L \oplus E \cong \mathcal{O}^{N}$, we can define $P$ for $\tilde{U}$. Since $\tilde{U} \rightarrow U$ is an $\mathbf{A}^{1}$-weak equivalence this means that we have a well defined operation $P$ for any $G, r: G \rightarrow S_{n}$ and any quasi-projective $U$ with a free $G$-action. The following lemma is straightforward.

Lemma 5.5 Let $G$ be a finite group and $r: G \rightarrow S_{n}$ a permutational representation of $G$. Let $U, V$ be smooth quasi-projective schemes with free actions of $G$ and $f: U \rightarrow V$ an equivariant morphism. Then for any $x$ one has $P_{U}(x)=f^{*}\left(P_{V}(x)\right)$.

Lemma 5.6 Let $G, r, U, L, \phi$ be as above. Then the following diagram of morphisms of pointed sheaves commutes

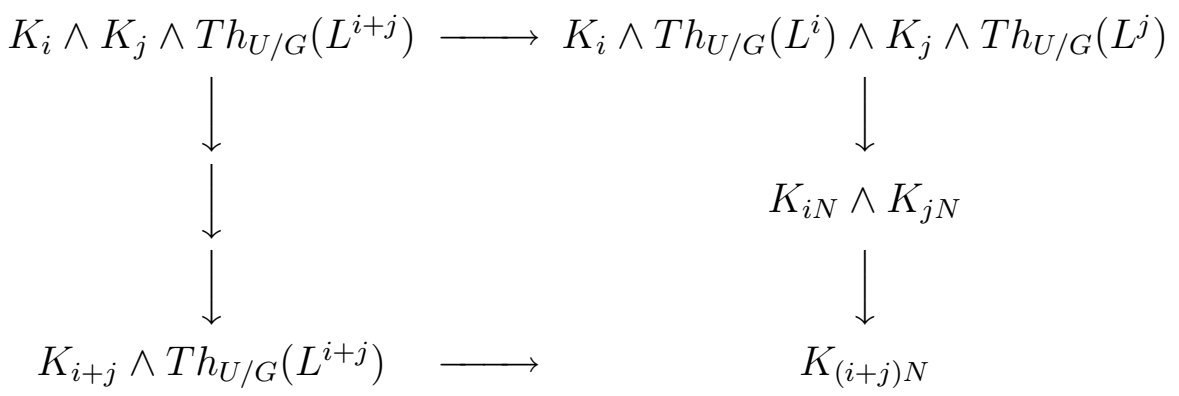


Proof: Direct comparison.

Lemma 5.7 For $a \in \tilde{H}^{2 i, i}\left(F_{\bullet}\right)$ and $b \in \tilde{H}^{2 j, j}\left(F_{\bullet}^{\prime}\right)$ one has

$$
P(a \wedge b)=\Delta^{*}(P(a) \wedge P(b))
$$

where $\Delta: U / G \rightarrow U / G \times U / G$ is the diagonal.

Proof: By Lemma 5.3 we have $\tilde{P}(a \wedge b)=\delta^{*}(\tilde{P}(a) \wedge \tilde{P}(b))$ where

$$
\delta: T h_{U / G}\left(L^{i+j}\right) \rightarrow T h_{U / G}\left(L^{i}\right) \wedge T h_{U / G}\left(L^{j}\right)
$$

Therefore by (5.2):

$$
P(a \wedge b) t_{L^{i+j}}=\delta^{*}\left(\left(P(a) t_{L^{i}}\right) \wedge\left(P(b) t_{L^{j}}\right)\right)
$$

Since $\delta^{*}\left(t_{L^{i}} \wedge t_{L^{j}}\right)=t_{L^{i+j}}$ the Thom isomorphism theorem implies (5.4).

Lemma 5.8 Let $\eta^{i} \in \tilde{H}^{2 i, i}\left(T^{i}\right)$ be the tautological class. Then $P\left(\eta^{i}\right)=$ $\delta^{*}\left(t_{\xi_{n}^{i}}\right)$ where $\xi_{n}$ is the vector bundle on $U / G$ corresponding to the representation $r: G \rightarrow S_{n}$ and $\delta$ is the map on Thom spaces defined by the embedding of vector bundles $\mathcal{O}^{i} \rightarrow \xi_{n}^{i}$ on $U / G$.

Proof: Take $\mathbf{A}^{i} / \mathbf{A}^{i}-\{0\}$ as a model of $T^{i}$ such that $\eta^{i}$ is given by the tautological section $Z$ of $\mathbf{Z}_{t r}\left(\mathbf{A}^{i}\right)$ on $\mathbf{A}^{i}$. Applying the construction of $\tilde{P}$ to the corresponding diagonal cycle $Z$ we get the restriction to $T h\left(L^{i}\right)$ of the morphism

$$
W: T h\left(L^{i} \oplus \xi_{n}^{i}\right) \rightarrow \mathbf{Z}_{t r}\left(\mathbf{A}^{N} i\right)
$$

corresponding, by Construction 5.1, to the tautological cycle on $\xi_{n}^{2 i}$ over $\xi_{n}^{i}$. In view of Corollary $4.4 W$ represents the Thom class of the trivial bundle $\left(L \oplus \xi_{n}\right)^{i}$. Applying Thom isomorphism to get $P(Z)$ we conclude that $P(Z)$ is the restriction of the Thom class of $T h\left(\xi_{n}^{i}\right)$ with respect to the morphism $\delta$.

Lemma 5.9 Let $E$ be a vector bundle on $X$. Then $P\left(t_{E}\right)=\delta^{*}\left(t_{E \otimes \xi_{n}}\right)$ where $\xi_{n}$ is the vector bundle on $U / G$ corresponding to the representation $r: G \rightarrow$ $S_{n}$ and $\delta$ is the map on Thom spaces defined by the embedding of vector bundles $E \otimes \mathcal{O} \rightarrow E \otimes \xi_{n}$ on $X \times(U / G)$. 
Proof: Follows from Corollary 4.4 and Lemma 5.8.

Let $*$ be a $k$-point of $U / G$ which lifts to a $k$-point of $U$. Let $i: S^{0} \rightarrow(U / G)_{+}$ be the corresponding morphism. The following lemma is straightforward.

Lemma 5.10 The composition

$$
K_{i, R} \stackrel{I d \wedge i}{\rightarrow} K_{i, R} \wedge(U / G)_{+} \stackrel{P}{\rightarrow} K_{i n, R}
$$

coincides in $H_{\bullet}$ with the $n$-th power map.

Proof: Let $L_{*}$ be the fiber of $L$ over our distinguished point $*$. Then, if we compute the analog of our composition using $\tilde{P}$ instead of $P$, we get the map $K_{i, R} \wedge T h\left(L_{*}\right) \rightarrow K_{i N, R}$ which is of the form $a\left(Z^{\otimes n}\right)$ where $Z$ is the tautological cycle on $K_{i, R}$ and $a(Z)$ is Construction 5.1 with respect to the isomorphism $\left(\xi_{n}\right)_{*}^{\oplus i} \oplus L_{*}^{\oplus i} \rightarrow \mathcal{O}^{i N}$. Our result follows now from Lemma 5.2 since $\left(\xi_{n}\right)_{*}=\mathcal{O}^{n}$ is the trivial bundle.

\section{Motivic cohomology of $B \mu_{l}$ and $B S_{l}$}

Let $G$ be a linear algebraic group and $G \rightarrow G L(V)$ a faithful representation of $G$. Denote by $\tilde{V}_{n}$ the open subset in $\mathbf{A}(V)^{n}$ where $G$ acts freely. We have a sequence of closed embeddings $\tilde{V}_{n} \rightarrow \tilde{V}_{n+1}$ given by $\left(v_{1}, \ldots, v_{n}\right) \mapsto$ $\left(v_{1}, \ldots, v_{n}, 0\right)$. Set $B G=\operatorname{colim}_{n} \tilde{V}_{n} / G$ where $\tilde{V}_{n} / G$ is the quotient scheme and the colimit is taken in the category of sheaves. In [6] we used the notation $B_{g m} G$ for $B G$. As shown there, the homotopy type of $B G$ does not depend on the choice of $G \rightarrow G L(V)$. We denote by $*$ any $k$-rational point of $B G$ which lifts to a $k$-rational point in one of the $\tilde{V}_{n}$ 's. The goal of this section is to describe motivic cohomology of $B S_{l}$ with coefficients in $\mathbf{Z} / l$. We start with the following general result.

Proposition 6.1 For any $G$ and $V$ as above the map

$$
i_{n}: \tilde{V}_{n} / G \rightarrow \tilde{V}_{n+1} / G
$$

defines an isomorphism on motivic cohomology of weigh less than $n$.

Proof: The morphism $\tilde{V}_{n} / G \rightarrow \tilde{V}_{n+1} / G$ can be represented by the composition

$$
\tilde{V}_{n} / G \rightarrow\left(\tilde{V}_{n} \times V\right) / G \rightarrow \tilde{V}_{n+1} / G
$$


The first of these maps is the zero section of a vector bundle $\left(\tilde{V}_{n} \times V\right) / G \rightarrow$ $\tilde{V}_{n} / G$ and gives an isomorphism on motivic cohomology by homotopy invariance. The second map is an open embedding of smooth schemes and the codimension of the complement is at least $n$. By Lemma 3.6 it defines an isomorphism on $\tilde{H}^{*,<n}$.

Corollary 6.2 One has

$$
H^{*, *}(B G)=\lim _{n} H^{*, *}\left(\tilde{V}_{m} / G\right)
$$

Proof: Given any sequence of maps of pointed simplicial sheaves $F_{\bullet, n} \rightarrow$ $F_{\bullet, n+1}$ with the colimit $F_{\bullet, \infty}$ we have a long exact sequence of the form

$$
\rightarrow \prod \tilde{H}^{*-1, *}\left(F_{\bullet, n}\right) \rightarrow \tilde{H}^{*, *}\left(F_{\bullet, \infty}\right) \rightarrow \prod \tilde{H}^{*, *}\left(F_{\bullet, n}\right) \rightarrow \prod \tilde{H}^{*, *}\left(F_{\bullet, n}\right) \rightarrow
$$

The limit $\lim _{n} \tilde{H}^{*, *}\left(F_{\bullet}, n\right)$ is the kernel of the fourth arrow and therefore to prove the corollary it is sufficient to show that the map

$$
I d-\prod i_{n}^{*}: \prod H^{*, *}\left(\tilde{V}_{n} / G\right) \rightarrow \prod H^{*, *}\left(\tilde{V}_{n} / G\right)
$$

is an epimorphism. It follows from the proposition.

Denote by $\mu_{l}$ the groups scheme of l-th roots of unity

$$
\mu_{l}:=\operatorname{ker}\left(\mathbf{G}_{m} \stackrel{z^{l}}{\rightarrow} \mathbf{G}_{m}\right)
$$

Lemma 6.3 Let $B \mu_{l}$ be defined with respect to the tautological 1-dimensional representation of $\mu_{l}$. Then one has

$$
B \mu_{l}=\mathcal{O}(-l)_{\mathbf{P}^{\infty}}-z\left(\mathbf{P}^{\infty}\right)
$$

Proof: We have $\tilde{V}_{n}=\mathbf{A}^{n}-\{0\}$. The projection $\mathbf{A}^{n}-\{0\} \rightarrow \mathbf{P}^{n-1}$ is invariant under the action of $\mu_{l}$ and therefore gives a map $\left(\mathbf{A}^{n}-\{0\}\right) / \mu_{l} \rightarrow \mathbf{P}^{n-1}$. One verifies that this map is isomorphic to the complement to the zero section of the line bundle $\mathcal{O}(-l)$ on $\mathbf{P}^{n-1}$.

Let $r: G \rightarrow G L(V)$ be a linear representation of $G$. It defines an action of $G$ on the affine space

$$
\mathbf{A}(V)=\operatorname{Spec}\left(S^{\bullet} V^{*}\right)
$$

corresponding to $V$. If $U$ is a scheme with a free action of $G$ then the projection $(\mathbf{A}(V) \times U) / G \rightarrow U / G$ is a vector bundle. We say that this is the vector bundle defined by $r$. 
Lemma 6.4 The line bundle on $B \mu_{l}$ defined by the tautological representation of $\mu_{l}$ is isomorphic with respect to (6.1) to the pull-back of $\mathcal{O}(1)$.

Proof: Let $L=\mathbf{A}^{1}$ with the standard action of $\mathbf{G}_{m}$ and the corresponding action of $\mu_{l}$. The square

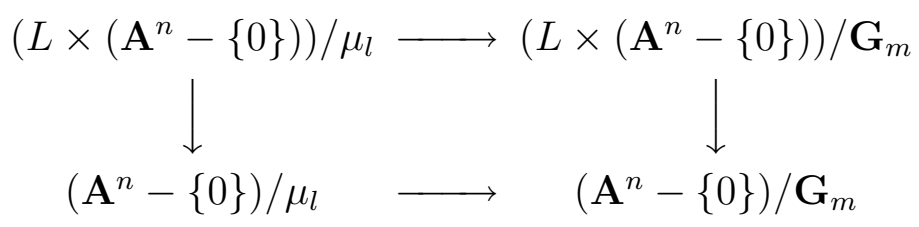

is pull-back. The fact that the right vertical arrow is $\mathcal{O}(1) \rightarrow \mathbf{P}^{n-1}$ is standard (e.g. it is not $\mathcal{O}(-1)$ because it has a section other than the zero one).

Lemma 6.3 implies that one has a cofibration sequence of the form

$$
\left(B \mu_{l}\right)_{+} \rightarrow\left(\mathcal{O}(-l)_{\mathbf{P}^{\infty}}\right)_{+} \rightarrow \operatorname{Th}(\mathcal{O}(-l))
$$

For a vector bundle $E$ of dimension $d$, the composition of the Thom isomorphism $H^{*, *}(X) \rightarrow \tilde{H}^{*+2 d, *+d}(T h(E))$ with the restriction to the zero section $z^{*}: \tilde{H}^{*, *}(T h(E)) \rightarrow H^{*, *}(X)$ is given by

$$
x \mapsto z^{*}\left(x t_{E}\right)=x z^{*}\left(t_{E}\right)=x e(E)
$$

By Lemma 4.5, $e(\mathcal{O}(-l))=l \sigma$ where $\sigma \in H^{2,1}\left(\mathbf{P}^{\infty}\right)$ is the same class as in the projective bundle theorem 4.1. Therefore, the long exact sequence defined by (6.2) is of the form

$$
\ldots \rightarrow H^{*-2, *-1}[[\sigma]] \stackrel{l \sigma}{\rightarrow} H^{*, *}[[\sigma]] \rightarrow H^{*, *}\left(B \mu_{l}\right) \rightarrow H^{*-1, *-1}[[\sigma]] \rightarrow \ldots
$$

The short exact sequence of abelian groups

$$
0 \rightarrow \mathbf{Z} \rightarrow \mathbf{Z} \rightarrow \mathbf{Z} / l \rightarrow 0
$$

defines a homomorphism

$$
\delta: \tilde{H}^{*, *}(-, \mathbf{Z} / l) \rightarrow \tilde{H}^{*+1, *}(-, \mathbf{Z})
$$

Let $v$ be Euler class of the line bundle on $B \mu_{l}$ corresponding to the tautological representation of $\mu_{l}$. 
Lemma 6.5 There exists a unique element $u \in H^{1,1}\left(B \mu_{l}, \mathbf{Z} / l\right)$ such that the restriction of $u$ to $*$ is zero and $\delta(u)=v$.

Proof: Existence follows from the exact sequence

$$
H^{1,1}\left(B \mu_{l}, \mathbf{Z}\right) \rightarrow H^{1,1}\left(B \mu_{l}, \mathbf{Z} / l\right) \rightarrow H^{2,1}\left(B \mu_{l}, \mathbf{Z}\right) \stackrel{l}{\rightarrow} H^{2,1}\left(B \mu_{l}, \mathbf{Z}\right)
$$

and the fact that

$$
l v=-l e(\mathcal{O}(-1))=-e(\mathcal{O}(-l))=0
$$

in $H^{2,1}\left(B \mu_{l}, \mathbf{Z}\right)$. The exact sequence (6.3) around $H^{1,1}\left(B \mu_{l}, \mathbf{Z}\right)$ shows that $H^{1,1}\left(B \mu_{l}, \mathbf{Z}\right)=H^{1,1}(k)$. This implies the uniqueness.

Proposition 6.6 For any pointed simplicial sheaf $F_{\text {. }}$ the elements $v^{i}$ and $u v^{i}, i \geq 0$ form a basis of $\tilde{H}^{*, *}\left(F_{\bullet} \wedge\left(B \mu_{l}\right)_{+}\right)$over $\tilde{H}^{*, *}\left(F_{\bullet}\right)$.

Proof: The standard argument shows that it is sufficient to consider the case when $F_{\bullet}$ is of the form $X_{+}$for a smooth scheme $X$. The same reasoning as we used to establish (6.3) applies to the motivic cohomology groups of $X \times B \mu_{l}$ for any smooth scheme $X$ and we get the following result.

Lemma 6.7 For any smooth scheme $X$ there is a long exact sequence of $H^{*, *}(X)[[\sigma]]$-modules of the form

$$
\begin{gathered}
\ldots \rightarrow H^{*-2, *-1}(X)[[\sigma]] \stackrel{l \sigma}{\rightarrow} H^{*, *}(X)[[\sigma]] \rightarrow H^{*, *}\left(X \times B \mu_{l}\right) \rightarrow \\
\rightarrow H^{*-1, *-1}(X)[[\sigma]] \rightarrow \ldots
\end{gathered}
$$

For $\mathbf{Z} / l$-coefficients we have $l \sigma=0$ and (6.4) becomes a short exact sequence of $H^{*, *}(X)[[\sigma]]$-modules of the form

$$
0 \rightarrow H^{*, *}(X)[[\sigma]] \rightarrow H^{*, *}\left(X \times B \mu_{l}\right) \rightarrow H^{*-1, *-1}(X)[[\sigma]] \rightarrow 0
$$

Let $u^{\prime}$ be an element in $H^{1,1}\left(B \mu_{l}, \mathbf{Z} / l\right)$ such that the image of $u^{\prime}$ in

$$
H^{0,0}(\operatorname{Spec}(k))=\mathbf{Z} / l
$$

is 1 and the restriction of $u^{\prime}$ to $X \times *$ is zero. Since $v$ is the image of $\sigma$, the short exact sequence (6.5) implies that the monomials $u^{\prime} v^{i}$ and $v^{i}$ form a basis of $H^{*, *}\left(X \times B \mu_{l}\right)$ over $H^{*, *}(X)$. On the other hand, the image of $u$ 
in $H^{0,0}(\operatorname{Spec}(k))=\mathbf{Z} / l$ is not zero and hence $u=c u^{\prime}$ where $c \in(\mathbf{Z} / l)^{*}$. This implies that the monomials $u v^{i}, v^{i}$ also form a basis.

To describe the multiplicative structure of $\tilde{H}^{*, *}\left(F_{\bullet} \wedge\left(B \mu_{l}\right)_{+}\right)$it is sufficient to find an expression for $u^{2}$. If $l \neq 2$ then $u^{2}=0$ since the multiplication in motivic cohomology is graded commutative. Consider the case $l=2$. We can clearly assume that $F_{\bullet}=\operatorname{Spec}(k)_{+}$. The element $u^{2}$ lies in $H^{2,2}$ and Lemma 6.6 shows that

$$
H^{2,2}\left(B \mu_{l}\right)=H^{0,1}(k) v \oplus H^{1,1}(k) u \oplus H^{2,2}(k)
$$

Since $u$ is zero in $*$ the projection of $u^{2}$ to the last factor is zero and we get $u^{2}=x v+y u$ for $x \in H^{0,1}(k)$ and $y \in H^{1,1}(k)$. To compute $y$ consider the map

$$
\mathbf{A}^{1}-\{0\}=\left(\mathbf{A}^{1}-\{0\}\right) / \mu_{l} \rightarrow B \mu_{l}
$$

If we choose the distinguished point of $B \mu_{l}$ to be the image of the point $(1,0, \ldots)$, this map is the embedding of the fiber of the line bundle $\mathcal{O}(-l)-$ $z\left(\mathbf{P}^{\infty}\right) \rightarrow \mathbf{P}^{\infty}$ which contains the distinguished point. The pull-back along this map coincides with the composition of the last map of (6.3) with the map $H^{*, *}[[\sigma]] \rightarrow \tilde{H}^{*, *}\left(\mathbf{A}^{1}-\{0\}, 1\right)$ which sends 1 to the generator of $\tilde{H}^{1,1}\left(\mathbf{A}^{1}-\right.$ $\{0\}, 1)$ and $\sigma$ to zero. In particular, the pull-back of $u$ along (6.6) is non-zero. The following lemma implies now that $y=\rho$ where $\rho$ is the class of -1 in $H^{1,1}$.

Lemma 6.8 Let $w$ be the non zero element of

$$
\tilde{H}^{1,1}\left(\left(\mathbf{A}^{1}-\{0\}, 1\right), \mathbf{Z} / 2\right)=\mathbf{Z} / 2 .
$$

Then $w^{2}=\rho w$.

Proof: We need to compare two motivic cohomology classes in $H^{2,2}\left(\mathbf{A}^{1}-\right.$ $\{0\})$. Let $\operatorname{Spec}(k(t)) \rightarrow \mathbf{A}^{1}-\{0\}$ be the embedding of the generic point. Since the base field $k$ may be assumed to be perfect, the Gysin long exact sequence in motivic cohomology implies that the kernel of the induced map in $H^{2,2}$ is covered by a direct sum of groups of the form $H^{0,1}(\operatorname{Spec}(E), \mathbf{Z})$. Since such groups are zero it is a monomorphism. Therefore, it is sufficient to show that $t t=\rho t$ in $H^{2,2}(\operatorname{Spec}(k(t)), \mathbf{Z})$. By [8], this group is isomorphic to $K_{2}^{M}(k(t))$ and we conclude by the well known relation $(a, a)=(-1, a)$ in the Milnor's K-theory. 
To compute $x$ note that $H^{0,1}(k, \mathbf{Z} / 2)=\mu_{2}(k)$. If $\operatorname{char}(k)=2$ then this group is zero. If $\operatorname{char}(k) \neq 2$ it is $\mathbf{Z} / 2$ and we only need to know whether $x$ is zero or not. The following lemma implies that $x=\tau$ is the generator of $\mathbf{Z} / 2$.

Lemma 6.9 Let $k$ be a separably closed field of characteristic not equal to 2. Then $u^{2} \neq 0$.

Proof: We have a natural transformation from the motivic cohomology to the etale cohomology with $\mathbf{Z} / 2$-coefficients. For a class $u$ in the etale $H^{1}$ we have $u^{2}=\beta(u)$ where $\beta$ is the Bockstein homomorphism. Since $k$ is separably closed and in particular contains $\sqrt{-1}$, the Bockstein in the etale cohomology commutes with the Bockstein in the motivic cohomology and we conclude that the image of $u^{2}$ in the etale cohomology coincides with the image of $v=\beta(u)$ in the etale cohomology. An etale analog of the long exact sequence (6.3) shows that the image of $v$ in the etale cohomology is non zero.

We proved the following result.

Theorem 6.10 For any field $k$ and a pointed simplicial sheaf $F_{\bullet}$ over $k$ one has:

$$
\tilde{H}^{*, *}\left(F_{\bullet} \wedge\left(B \mu_{l}\right)_{+}, \mathbf{Z} / l\right)=\tilde{H}^{*, *}\left(F_{\bullet}, \mathbf{Z} / l\right)[[u, v]] /\left(u^{2}=\tau v+\rho u\right)
$$

where:

1. $\rho$ is the class of -1 in $H^{1,1}(k)$

2. $\tau$ is zero if $l \neq 2$ or $\operatorname{char}(k)=2$

3. $\tau$ is the generator of $H^{0,1}(k, \mathbf{Z} / 2)=\mu_{2}(k)$ if $l=2$ and $\operatorname{char}(k) \neq 2$.

We also need the following additional fact about cohomology of $B \mu_{l}$.

Lemma 6.11 Let $c \in \operatorname{Aut}\left(\mu_{\mathbf{l}}\right)=(\mathbf{Z} / l)^{*}$ and let $\underline{c}$ be the corresponding automorphism of $B \mu_{l}$. Then $\underline{\underline{c}}^{*}(u)=c u$ and $\underline{c}^{*}(v)=c v$.

Proof: Let $L$ be the line bundle on $B \mu_{l}$ corresponding to the tautological 1-dimensional representation $\lambda$ of $\mu_{l}$. Then $v$ is the Euler class of $L$ and $u$ is the only element in $H^{1,1}(-, \mathbf{Z} / l)$ which is zero at $*$ and which maps to $v$ under the map $\delta: H^{1,1}(-, \mathbf{Z} / l) \rightarrow H^{2,1}(-, \mathbf{Z})$. The automorphism $\underline{c}$ takes the tautological 1-dimensional representation $\lambda$ to $\lambda^{\otimes c}$ and, therefore, it takes $L$ to $L^{\otimes c}$. Our result follows now from Lemma 4.5. 
Our next goal is to compute $\tilde{H}^{*, *}\left(F_{\bullet} \wedge\left(B S_{l}\right)_{+}, \mathbf{Z} / l\right)$ where $S_{l}$ is the symmetric group and $l$ is a prime not equal to $\operatorname{char}(k)$.

Lemma 6.12 Let $G$ be a finite group and $H$ a subgroup of $G$. Assume that $[G: H]$ is invertible in the coefficients ring $R$. Then, for any pointed simplicial sheaf $F_{\bullet}$, the map of motivic cohomology

$$
\tilde{H}^{*, *}\left(F_{\bullet} \wedge B G_{+}, R\right) \rightarrow \tilde{H}^{*, *}\left(F_{\bullet} \wedge B H_{+}, R\right)
$$

is a split mono and its image is contained in the invariants under the action of the normalizer of $H$ in $G$.

Proof: We will use the notations established at the beginning of Section 6 . Choose a linear representation $G \rightarrow G L(V)$. The map (6.8) is defined by the collection of maps $p_{n}: \tilde{V}_{n} / H \rightarrow \tilde{V}_{n} / G$ with respect to the identification of Corollary 6.2. The maps $p_{n}$ are finite etale of degree $[G: H]$ and the fundamental cycle on $\tilde{V}_{n} / H$ over $\tilde{V}_{n} / G$ defines a map of freely generated sheaves with transfers

$$
p^{\#}: \mathbf{Z}_{t r}\left(\tilde{V}_{n} / G\right) \rightarrow \mathbf{Z}_{t r}\left(\tilde{V}_{n} / H\right)
$$

The composition of $p^{\#} \mathbf{Z}_{t r}(p)$ is the multiplication by $[G: H]$. Replacing $F$. by its the standard simplicial resolution by coproducts of representable sheaves we may assume that terms of $F$. are coproducts of sheaves of the form $\left(h_{U}\right)_{+}$. Then,

$$
\tilde{H}^{p, q}\left(F_{\bullet} \wedge X_{+}, A\right)=H_{o m}\left(N\left(\mathbf{Z}_{t r}\left(F_{\bullet}\right)\right) \otimes \mathbf{Z}_{t r}(X), A(q)[p]\right)
$$

where $N(-)$ is the normalized chain complex functor from simplicial sheaves with transfers to complexes of sheaves with transfers. In particular these groups are functorial in $\mathbf{Z}_{t r}(-)$ which implies the first claim of the proposition.

The second claim follows from the fact that the normalizer of $H$ in $G$ acts on $\tilde{V}_{n} / H$ over $\tilde{V}_{n} / G$ and the map $p^{\#}$, being defined by the fundamental cycle, is invariant under this action.

Let $\xi_{l}$ be the vector bundle on $B S_{l}$ corresponding to the tautological permutational representation. Then we have a monomorphism $\mathcal{O} \rightarrow \xi_{l}$ and the quotient $\xi_{l} / \mathcal{O}$ is again a vector bundle. Let

$$
d:=e\left(\xi_{l} / \mathcal{O}\right)
$$


Assume that $l$ is a prime different from $\operatorname{char}(k)$ and that there exists a primitive l-th root of unity $\zeta$ in $k$. The choice of $\zeta$ defines a weak equivalence $B \mu_{l} \rightarrow B \mathbf{Z} / l$ and the inclusion $\mathbf{Z} / l \rightarrow S_{l}$ gives a map

$$
p_{\zeta}: B \mu_{l} \rightarrow B \mathbf{Z} / l \rightarrow B S_{l}
$$

Lemma 6.13 One has:

$$
p_{\zeta}^{*}(d)=-u^{l-1}
$$

Proof: The element $p_{\zeta}^{*}(d)$ is the Euler class of $\xi / \mathcal{O}$ where $\xi$ corresponds to the regular representation of $\mathbf{Z} / l$ under our isomorphism $\mathbf{Z} / l \rightarrow \mu_{l}$. Therefore, we have $\xi=\oplus_{i=0}^{l-1} L^{i}$ where $L$ is the line bundle corresponding to the tautological 1-dimensional representation of $\mu_{l}$. By Lemma 4.6 and Lemma 4.5 we get

$$
p_{\zeta}^{*}(d)=\prod_{i=1}^{l-1}(i u)=u^{l-1}
$$

Theorem 6.14 Let $l$ be a prime and $k$ a field of characteristic not equal to l. There exists a unique class $c \in H^{2 l-3, l-1}\left(B S_{l}, \mathbf{Z} / l\right)$ such that $\delta(c)=d$ and the restriction of $c$ to $*$ is zero.

Proof: For $l=2$ we have $S_{2}=\mathbf{Z} / 2=\mu_{2}$ and our result follows from Lemma 6.5. Assume that $l>2$. The transfer argument shows that to prove the theorem for $k$ it is sufficient to prove it for a separable extension of $k$ of degree prime to $l$. In particular, we may assume that $k$ contains a primitive $l$-th root of unity $\zeta$.

To prove the existence of $c$ we need to show that $d$ is an $l$-torsion element in $H^{*, *}(-, \mathbf{Z})$. For any ring of coefficients where $(l-1)$ ! is invertible, the map $p_{\zeta}$ defines, by Lemma 6.12 , a split injection:

$$
H^{*, *}\left(B S_{l}\right) \rightarrow H^{*, *}\left(B \mu_{l}\right)^{A u t\left(\mu_{l}\right)}
$$

In particular, since (6.10) is an injection for coefficients in $\mathbf{Z}$ localized at $l$, it is sufficient to prove that the image of $d$ in $H^{*, *}\left(B \mu_{l}, \mathbf{Z}\right)$ is an l-torsion element. This follows from Lemma 6.13.

To show that $c$ is unique, it is sufficient to check that the map

$$
\delta: H^{2 l-3, l-1}\left(B S_{l}, \mathbf{Z} / l\right) \rightarrow H^{2 l-2, l-1}\left(B S_{l}, \mathbf{Z}\right)
$$


is injective. Injectivity of (6.10) for $\mathbf{Z} /$-coefficients implies that it is sufficient to show that the map

$$
\delta: H^{2 l-3, l-1}\left(B \mu_{l}, \mathbf{Z} / l\right)^{A u t\left(\mu_{l}\right)} \rightarrow H^{2 l-2, l-1}\left(B \mu_{l}, \mathbf{Z}\right)^{A u t\left(\mu_{l}\right)}
$$

is injective. Lemma 6.11 implies that for $\mathbf{Z} / l$-coefficients the right hand side of $(6.10)$ is of the form

$$
H^{*, *}\left(B \mu_{l}, \mathbf{Z} / l\right)^{A u t\left(\mu_{l}\right)}=H^{*, *}[[x, y]] /\left(x^{2}=0\right)
$$

where $x=v u^{l-2}$ and $y=u^{l-1}$. This descriptions shows that

$$
H^{2 l-3, l-1}\left(B S_{l}, \mathbf{Z} / l\right)^{A u t\left(\mu_{l}\right)}=\mathbf{Z} / l
$$

generated by $x$. We have $\delta(x)=u^{l-1} \neq 0$. Therefore, (6.11) is injective.

Lemma 6.15 Let $l$ be a prime such that $\operatorname{char}(k) \neq l$ and $\zeta$ be an $l$-th root of unity in $k$. Let further $p_{\zeta}: B \mu_{l} \rightarrow B S_{l}$ be the morphism defined by $\zeta$. Then one has:

$$
p_{\zeta}^{*}(c)=-v u^{l-2}
$$

Proof: We may assume that $l>2$. Then, the description (6.12) implies that $p_{\zeta}^{*}(c)=a v u^{l-2}$ for an element $a \in(\mathbf{Z} / l)^{*}$. Since

$$
\delta\left(p_{\zeta}^{*}(c)\right)=p_{\zeta}(\delta(c))=p_{\zeta}(d)=-u^{l-1}
$$

and

$$
\delta\left(v u^{l-2}\right)=u^{l-1}
$$

we conclude that $a=-1$.

Theorem 6.16 For any pointed simplicial sheaf $F_{\text {• over }} k$ one has:

$$
\tilde{H}^{*, *}\left(F_{\bullet} \wedge\left(B S_{l}\right)_{+}, \mathbf{Z} / l\right)= \begin{cases}\tilde{H}^{*, *}\left(F_{\bullet}, \mathbf{Z} / l\right)[[c, d]] /\left(c^{2}=\tau d+\rho c\right) & \text { for } l=2 \\ \tilde{H}^{*, *}\left(F_{\bullet}, \mathbf{Z} / l\right)[[c, d]] /\left(c^{2}=0\right) & \text { for } l \neq 2\end{cases}
$$

where:

1. $\rho$ is the class of -1 in $H^{1,1}(k)$

2. $\tau$ is the generator of $H^{0,1}(k, \mathbf{Z} / 2)=\mu_{2}(k)$. 
Proof: For $l=2$ we have $S_{2}=\mathbf{Z} / 2=\mu_{2}$ and our result follows from Theorem 6.10. Assume that $l>2$. We need to show that the map

$$
\tilde{H}^{*, *}\left(F_{\bullet}\right)[[c, d]] /\left(c^{2}=0\right) \rightarrow \tilde{H}^{*, *}\left(F_{\bullet} \wedge\left(B S_{l}\right)_{+}, \mathbf{Z} / l\right)
$$

is an isomorphism. By the transfer argument we may assume that $k$ contains a primitive l-th root of unity. By Lemma 6.8 the homomorphism

$$
\tilde{H}^{*, *}\left(F_{\bullet} \wedge\left(B S_{l}\right)_{+}, \mathbf{Z} / l\right) \rightarrow \tilde{H}^{*, *}\left(F_{\bullet} \wedge\left(B \mu_{l}\right)_{+}, \mathbf{Z} / l\right)^{A u t\left(\mu_{l}\right)}
$$

defined by a choice of a primitive root $\zeta$ is a mono. Therefore, it is sufficient to show that the composition

$$
\tilde{H}^{*, *}\left(F_{\bullet}\right)[[c, d]] /\left(c^{2}=0\right) \rightarrow \tilde{H}^{*, *}\left(F_{\bullet} \wedge\left(B S_{l}\right)_{+}\right) \rightarrow \tilde{H}^{*, *}\left(F_{\bullet} \wedge\left(B \mu_{l}\right)_{+}\right)^{\operatorname{Aut}\left(\mu_{l}\right)}
$$

is an isomorphism. The fact that (6.14) is an isomorphism follows from the analog of formula (6.12) for $\tilde{H}^{*, *}\left(F_{\bullet} \wedge\left(B \mu_{l}\right)_{+}\right)^{A u t\left(\mu_{l}\right)}$ and Lemmas 6.13, 6.15.

Lemma 6.17 Let $M$ be a line bundle and $l$ a prime not equal to char $(k)$. Then $P_{l}(e(M))=e(M)^{l}+e(M) d$.

Proof: By the transfer argument we may assume that $k$ contains a primitive l-th root of unity. In view of Lemma 6.12 it is sufficient to prove our equality in the motivic cohomology of $X \times B \mu_{l}$. By Lemma 5.9 we have $P(e(M))=$ $e\left(M \otimes \xi_{l}\right)$. The vector bundle $\xi_{l}$ restricted to $B \mu_{l}$ splits into the sum of line bundles $L^{0} \oplus L^{1} \oplus \cdots \oplus L^{l-1}$ where $L$ is the line bundle corresponding to the tautological 1-dimensional representation of $\mu_{l}$. By Lemmas 4.6 and 4.5 we get:

$e\left(M \otimes \xi_{l}\right)=\prod_{i=0}^{l-1} e\left(M \otimes L^{i}\right)=\prod_{i}(e(M)+i e(L))=e(M)\left(e(M)^{l-1}-e(L)^{l-1}\right)$

Since the restriction of $d$ to $B \mu_{l}$ is $-e(L)^{l-1}$ (by Lemma 6.13) this finishes the proof.

\section{Symmetry theorem}

Let $G_{1}, G_{2}$ be two finite groups acting freely on $U_{1}$ and $U_{2}$ respectively. Let further $r_{i}: G_{i} \rightarrow S_{n_{i}}$ be permutational representations of $G_{i}, i=1,2, \xi_{i}$ the 
corresponding vector bundles on $U_{i} / G_{i}$ of dimension $n_{i}$ and $L_{i} \oplus \xi_{i} \rightarrow \mathcal{O}^{N_{i}}$ inverses of $\xi_{i}$. Consider the action of $G_{1} \times G_{2}$ on $U_{1} \times U_{2}$ and let $\xi_{1} \otimes \xi_{2}$ be the vector bundle on $\left(U_{1} \times U_{2}\right) /\left(G_{1} \times G_{2}\right)$ corresponding to $r_{1} \times r_{2}$. Consider the vector bundle

$$
L_{12}=\left(L_{1} \otimes \xi_{2}\right) \oplus L_{2}^{N_{1}}
$$

Then

$$
L_{12} \oplus\left(\xi_{1} \otimes \xi_{2}\right)=\left(\left(L_{1} \oplus \xi_{1}\right) \otimes \xi_{2}\right) \oplus L_{2}^{N_{1}}=\xi_{2}^{N_{1}} \oplus L_{2}^{N_{1}}=\mathcal{O}^{N_{2} N_{1}}
$$

i.e. $L_{12}$ is an inverse of $\xi_{1} \otimes \xi_{2}$. The canonical map $\mathcal{O} \rightarrow \xi_{1}$ gives a monomorphism $i: L_{1} \oplus L_{2}^{N_{1}} \rightarrow L_{12}$.

Lemma 7.1 The following diagram of pointed sheaves commutes:

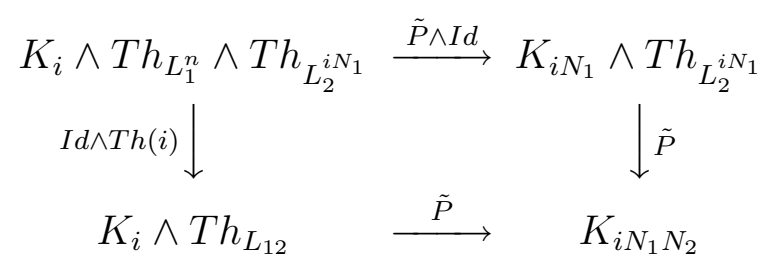

Proof: Direct comparison.

Proposition 7.2 Let $u$ be a class in $\tilde{H}^{2 i, i}\left(F_{\bullet}\right)$. Then:

$$
P_{2}\left(P_{1}(u)\right) e\left(\xi_{1} / \mathcal{O}\right)^{N_{1} i}=P_{12}(u) e\left(\xi_{1} / \mathcal{O}\right)^{N_{1} i}
$$

in $\tilde{H}^{2 i N_{1} N_{2}, i N_{1} N_{2}}\left(F_{\bullet} \wedge\left(U_{1} / G_{1} \times U_{2} / G_{2}\right)_{+}\right)$, where:

$$
\begin{gathered}
P_{i}=P_{G_{i}, r_{i}, U_{i}, L_{i}} \\
P_{12}=P_{G_{1} \times G_{2}, r_{1} \times r_{2}, U_{1} \times U_{2}, L_{12}}
\end{gathered}
$$

Proof: By Lemma 7.1 we have

$$
\tilde{P}_{2}\left(\tilde{P}_{1}(u)\right)=T h(i)^{*} \tilde{P}_{12}(u)
$$

or, equivalently,

$$
P_{2}\left(P_{1}(u) t_{L_{1}^{i}}\right) t_{L_{2}^{i N_{1}}}=T h(i)^{*}\left(P_{12}(u) t_{L_{12}}\right)
$$


By Lemma 4.7 we rewrite it as

$$
P_{2}\left(P_{1}(u) t_{L_{1}^{i}}\right) t_{L_{2}^{i N_{1}}}=P_{12}(u) e\left(\left(L_{1} \otimes\left(\xi_{2} / \mathcal{O}\right)\right)^{i}\right) t_{L_{1}^{i}} t_{L_{2}^{i N_{1}}}
$$

By Lemma 5.7 we get

$$
P_{2} P_{1}(u) P_{2}\left(t_{L_{1}^{i}}\right) t_{L_{2}^{i N_{1}}}=P_{12}(u) e\left(\left(L_{1} \otimes\left(\xi_{2} / \mathcal{O}\right)\right)^{i}\right) t_{L_{1}^{i}} t_{L_{2}^{i N_{1}}}
$$

By Lemmas 5.9 and 4.7 we get

$$
P_{2} P_{1}(u) e\left(\left(L_{1} \otimes\left(\xi_{2} / \mathcal{O}\right)\right)^{i}\right) t_{L_{1}^{i}} t_{L_{2}^{i N_{1}}}=P_{12}(u) e\left(\left(L_{1} \otimes\left(\xi_{2} / \mathcal{O}\right)\right)^{i}\right) t_{L_{1}^{i}} t_{L_{2}^{i N_{1}}}
$$

By Thom isomorphism 4.3, we get

$$
P_{2} P_{1}(u) e\left(\left(L_{1} \otimes\left(\xi_{2} / \mathcal{O}\right)\right)^{i}\right)=P_{12}(u) e\left(\left(L_{1} \otimes\left(\xi_{2} / \mathcal{O}\right)\right)^{i}\right)
$$

Multiplying both sides by $e\left(\left(\xi_{1} \otimes\left(\xi_{2} / \mathcal{O}\right)\right)^{i}\right)$ we get by Lemma 4.6

$$
P_{2} P_{1}(u) e\left(\xi_{2} / \mathcal{O}\right)^{i N_{1}}=P_{12}(u) e\left(\xi_{2} / \mathcal{O}\right)^{i N_{1}}
$$

Corollary 7.3 In the notations of the proposition assume that $r_{1}=l$ is a prime different from the characteristic of $k$. Then

$$
P_{2}\left(P_{1}(u)\right)=P_{12}(u)
$$

Proof: Replacing $U_{2}$ by an affine torsor we may assume that it is affine. Let $G_{2} \rightarrow G L(V)$ be the linear representation of $G_{2}$ corresponding to $r_{2}$ and $\tilde{V}_{m}$ the open subset of $\mathbf{A}\left(V^{\oplus m}\right)$ where $G$ acts freely. Then, for some $m$, there exists a $G_{2}$-equivariant map $U_{2} \rightarrow \tilde{V}_{m}$. By Lemma 5.5 it is sufficient to prove the corollary for $U_{2}=\tilde{V}_{m}$ and $G_{2}=S_{r_{2}}$. Proposition 7.2 together with Corollary 6.2 shows that we have

$$
P_{2}\left(P_{1}(u)\right) e\left(\xi_{1} / \mathcal{O}\right)^{N_{1} i}=P_{12}(u) e\left(\xi_{1} / \mathcal{O}\right)^{N_{1} i}
$$

on $F_{\bullet} \wedge\left(U_{1} / G_{1} \times B S_{l}\right)$. By Theorem 6.16 , multiplication with $e\left(\xi_{1} / \mathcal{O}\right)$ is injective and we conclude that $P_{2}\left(P_{1}(u)\right)=P_{12}(u)$.

Lemma 7.4 Let $U$ be a scheme with a free action of $G$ and let $r: G \rightarrow S_{n}$ be a permutational representation of $G$. Consider:

$$
P=P_{G \times G, r \times r, U \times U-\Delta(U)}: K_{i} \wedge((U \times U-\Delta(U)) /(G \times G))_{+} \rightarrow K_{i n^{2}}
$$

Then $P$ is invariant under the permutation of two copies of $U$. 
Proof: The action of $G \times G$ on $U \times U-\Delta(U)$ extends to a free action of the semidirect product $(G \times G) \propto \mathbf{Z} / 2$. The permutational representation $r \times r$ also extends to a permutational representation of $(G \times G) \propto \mathbf{Z} / 2$. Therefore, $P$ factors through the map

$K_{i} \wedge((U \times U-\Delta(U)) /(G \times G))_{+} \rightarrow K_{i} \wedge((U \times U-\Delta(U)) /(G \times G) \propto \mathbf{Z} / 2)_{+}$

which implies that it is symmetric.

Lemma 7.5 Let $X$ be a smooth variety and $Z$ a smooth subvariety in $Z$ of codimension $c$. Then for any pointed simplicial sheaf $F$ • the map

$$
\tilde{H}^{2 i, i}\left(F_{\bullet} \wedge X_{+}\right) \rightarrow \tilde{H}^{2 i, i}\left(F_{\bullet} \wedge(X-Z)_{+}\right)
$$

is an isomorphism for $i<c$.

Proof: Follows from Lemma 3.6.

Lemma 7.6 Under the assumptions of Lemma 7.4 the morphism

$$
P=P_{G \times G, r \times r, U \times U}: K_{i} \wedge(U \times U / G \times G)_{+} \rightarrow K_{i n^{2}}
$$

is invariant under the permutation of two copies of $U$.

Proof: Replacing $U$ by an affine torsor we may assume that it is affine. Let $G \rightarrow G L(V)$ be a faithful liner representation of $G$ and $\tilde{V}_{m}$ the open subset of $\mathbf{A}\left(V^{\oplus m}\right)$ where $G$ acts freely. Then, for some $m$, there exists a $G$-equivariant map $U \rightarrow \tilde{V}_{m}$. By Lemma 5.5 it is sufficient to show that $P$ is symmetric for $U=\tilde{V}_{m}$. This follows from Lemma 7.4 and Lemma 7.5 since we may choose $m$ such that the codimension of $\Delta\left(\tilde{V}_{m}\right)$ is larger than $i n^{2}$.

Corollary 6.2 together with Lemma 5.5 implies that there is a well defined morphism:

$$
P_{l}: K_{n} \wedge\left(B S_{l}\right)_{+} \rightarrow K_{n l}
$$

Theorem 7.7 The composition

$$
K_{i} \wedge\left(B S_{l}\right)_{+} \wedge\left(B S_{l}\right)_{+} \stackrel{P \wedge I d}{\rightarrow} K_{i l} \wedge\left(B S_{l}\right)_{+} \stackrel{P}{\rightarrow} K_{i l^{2}}
$$

is invariant under the permutation of two copies of $B S_{l}$. 
Proof: Let $\eta$ be the tautological motivic cohomology class of $K_{i}$. We need to show that $P(P(\eta))$ is invariant under the permutation of two copies of $B S_{l}$. Corollary 7.3 and Corollary 6.2 imply that $P(P(\eta))=P_{12}(\eta)$ where $P_{12}$ is the power operation corresponding to $S_{l} \times S_{l} \rightarrow S_{l^{2}}$. We conclude by Lemma 7.6 and, again, Corollary 6.2.

\section{Power operations and the Bockstein homomorphism}

We denote by $\beta$ the Bockstein homomorphism

$$
\tilde{H}^{*, *}(-, \mathbf{Z} / l) \rightarrow \tilde{H}^{*+1, *}(-, \mathbf{Z} / l)
$$

which is defined by the short exact sequence of the coefficients

$$
0 \rightarrow \mathrm{Z} / l \rightarrow \mathbf{Z} / l^{2} \rightarrow \mathbf{Z} / l \rightarrow 0
$$

It has the same properties as the Bockstein homomorphism in the ordinary cohomology. In particular, we have $\beta \beta=0$ and for $u \in \tilde{H}^{p, *}$,

$$
\beta(u v)=\beta(u) v+(-1)^{p} u \beta(v)
$$

The goal of this section is to prove Theorem 8.4. The method we use follows closely the method used to prove an analogous result in [7].

Let $U$ and $L$ be as in Construction 5.3. The proper push-forward of cycles defines a "transfer" map

$$
\operatorname{tr}: \underline{\operatorname{Hom}}\left(T h_{U}\left(L^{i}\right), K_{n, R}\right) \rightarrow \underline{\operatorname{Hom}}\left(T h_{U / G}\left(L^{n}\right), K_{n, R}\right)
$$

where $\underline{\operatorname{Hom}}(-,-)$ denotes the internal Hom-object in the category of pointed sheaves. Let $\mathbf{Z}_{(l)}$ be the local ring of $l$ in $\mathbf{Z}$. Denote by

$$
\Phi=\underline{\operatorname{Hom}}\left(T h_{U / G}\left(L^{n}\right), K_{n l, \mathbf{z}_{(l)}}\right) / \lim (t r)
$$

the pointed sheaf which corresponds to the quotient of the sheaf of abelian groups $\underline{\operatorname{Hom}}\left(T h_{U / G}\left(L^{n}\right), K_{n l, \mathbf{z}_{(l)}}\right)$ by the subgroup of elements of the form $l x$ where $x$ is in the image of the transfer map.

Lemma 8.1 The map of pointed sheaves

$$
K_{n, \mathbf{Z} / l} \rightarrow \underline{\operatorname{Hom}}\left(T h_{U / G}\left(L^{n}\right), K_{n l, \mathbf{Z} / l}\right)
$$

adjoint to the power operation $\tilde{P}_{l}$ lifts to a map of pointed sheaves

$$
K_{n, \mathbf{Z} / l} \rightarrow \Phi
$$


Proof: The pointed sheaf $K_{n, \mathbf{Z} / l}$ is a quotient sheaf of the sheaf $K_{n, \mathbf{Z}_{(l)}}$ and the power operation for integral coefficients defines a map

$$
K_{n, \mathbf{z}_{(l)}} \rightarrow \underline{\operatorname{Hom}}\left(T h_{U / G}\left(L^{n}\right), K_{n l, \mathbf{z}_{(l)}}\right)
$$

It is sufficient to show that the composition of this map with the projection to $\Phi$ factors through $K_{n, \mathbf{Z} / l}$ i.e. that for two cycles $Z_{1}, Z_{2}$ be cycles on $X \times \mathbf{A}^{n}$ with integral coefficients such that $Z_{1}-Z_{2}$ is divisible by $l$ the cycle $\tilde{P}_{l}\left(Z_{1}\right)-\tilde{P}_{l}\left(Z_{2}\right)$ is in $l \operatorname{Im}(t r)$. Let $Z_{i}^{\prime}$ be the cycle on $\left(X^{l} \times \mathbf{A}^{n l} \times U\right) / S_{l}$ whose pull-back to $X^{l} \times \mathbf{A}^{n l} \times U$ is $\left(p: X^{l} \times \mathbf{A}^{n l} \times U \rightarrow X^{l} \times \mathbf{A}^{n l}\right)^{*}\left(Z_{i}^{\otimes l}\right)$. It is sufficient to show that $Z_{1}^{\prime}-Z_{2}^{\prime}$ is in $\operatorname{lIm}\left(\pi_{*}\right)$ where

$$
\pi: X^{l} \times \mathbf{A}^{n l} \times U \rightarrow\left(X^{l} \times \mathbf{A}^{n l} \times U\right) / S_{l}
$$

is the projection or, equivalently, that

$$
p^{*}\left(Z_{1}^{\otimes l}\right)-p^{*}\left(Z_{2}^{\otimes l}\right)=l \pi^{*} \pi_{*}(Y)
$$

for some $Y$. We have $Z_{1}-Z_{2}=l W$ and the left hand side can be rewritten as $p^{*}\left(\left(Z_{2}+l W\right)^{\otimes l}\right)-p^{*}\left(Z_{2}^{\otimes l}\right)$. Since any $S_{l}$-invariant cycle with coefficients divisible by $l$ is of the form $\pi^{*} \pi_{*}(-)$ it is sufficient to consider the summands in this expression with coefficients not divisible by $l^{2}$. They are of the form $l\left(Z_{2} \otimes \ldots \otimes W \otimes \ldots \otimes Z_{2}\right)$. The sum of all such cycles is, up to multiplication by $(l-1)$ !, of the form $\pi^{*} \pi_{*}\left(W \otimes\left(Z_{2}\right)^{l-1}\right)$.

Lemma 8.2 Let $u$ be the tautological class in $\tilde{H}^{2 n, n}\left(K_{n, \mathbf{Z} / l}\right)$. Then $\beta \tilde{P}_{l}(u)$ lies in the image of the transfer map

$$
\tilde{H}^{2 n l, n l}\left(T h_{U}\left(L^{n}\right) \wedge K_{n, \mathbf{Z} / l}, \mathbf{Z} / l\right) \rightarrow \tilde{H}^{2 n l, n l}\left(T h_{U / G}\left(L^{n}\right) \wedge K_{n, \mathbf{Z} / l}, \mathbf{Z} / l\right)
$$

Proof: Consider the standard simplicial resolution $G_{\bullet} K_{n, R}$ (see Section 3). Since a cycle with $\mathbf{Z} /$-coefficients on a smooth scheme lifts to an integral cycle, the map

$$
G_{0} K_{n, \mathbf{Z} / l} \rightarrow \underline{\operatorname{Hom}}\left(T h_{U / G}\left(L^{n}\right), K_{n, \mathbf{Z} / l}\right)
$$

which represents $\tilde{P}_{l}(u)$ lifts to a map with values in $\underline{\operatorname{Hom}}\left(T h_{U / G}\left(L^{n}\right), K_{n, \mathbf{Z}}\right)$. Let $\phi$ be such a lifting. Then $\partial_{0} \phi-\partial_{1} \phi$ lands in $l K_{n, \mathbf{Z}}$ and $\left(\partial_{0} \phi-\partial_{1} \phi\right) / l$ gives a morphism of complexes $G_{*} K_{i, \mathbf{Z} / l} \rightarrow \underline{\operatorname{Hom}}\left(T h_{U / G}\left(L^{n}\right), K_{n, \mathbf{Z} / l}\right)$ which defines $\beta \tilde{P}_{l}(u)$. Lemma 8.1 implies immediately that $\left(\partial_{0} \phi-\partial_{1} \phi\right) / l$ lifts to a morphism with values in the image of the transfer map which implies the statement of the lemma. 
Lemma 8.3 For any $F_{\bullet}$, the transfer map in cohomology

$$
\tilde{H}^{*, *}\left(F_{\bullet} \wedge T h_{E S_{l}}\left(L^{n}\right), \mathbf{Z} / l\right) \rightarrow \tilde{H}^{*, *}\left(F_{\bullet} \wedge T h_{B S_{l}}\left(L^{n}\right), \mathbf{Z} / l\right)
$$

is zero.

Proof: The composition of the transfer map with the restriction map is the multiplication with the degree of the covering, in our case $l$ !. Hence it is sufficient to show that the restriction map

$$
\tilde{H}^{*, *}\left(F_{\bullet} \wedge T h_{B S_{l}}\left(L^{n}\right), \mathbf{Z} / l\right) \rightarrow \tilde{H}^{*, *}\left(F_{\bullet} \wedge T h_{E S_{l}}\left(L^{n}\right), \mathbf{Z} / l\right)
$$

is surjective. Since motivic cohomology of $E S_{l}$ are trivial and class on the right can be written as $u t$ where $u$ is in $\tilde{H}^{*, *}\left(F_{\bullet}\right)$ and $t$ is the Thom class. Any such ut is clearly in the image of the restriction map.

Theorem 8.4 For any $u \in \tilde{H}^{2 d, d}$ one has $\beta P(u)=0$.

Proof: By Lemma 8.2 and Lemma 8.3 we have $\beta(P(u) t)=\beta \tilde{P}(u)=0$. By (8.1) we get

$$
\beta(P(u) t)=\beta(P(u)) t
$$

and by the Thom isomorphism theorem we conclude that $\beta(P(u))=0$.

\section{Individual power operations: formulas}

In this section we assume that $l$ is a prime different from the characteristic of $k$. Let $w$ be a class in $\tilde{H}^{2 d, d}\left(F_{\bullet}, \mathbf{Z} / l\right)$. By Theorem 6.16 , the class $P_{l}(w)$ can be written uniquely as a linear combination of the form:

$$
P_{l}(u)=\sum_{i \geq 0} C_{i+1, d}(w) c d^{i}+D_{i, d}(w) d^{i}
$$

This defines cohomological operations:

$$
\begin{gathered}
C_{i, d}: \tilde{H}^{2 d, d}(-, \mathbf{Z} / l) \rightarrow \tilde{H}^{2 d+2(d-i)(l-1)+1, d+(d-i)(l-1)}(-, \mathbf{Z} / l) \\
D_{i, d}: \tilde{H}^{2 d, d}(-, \mathbf{Z} / l) \rightarrow \tilde{H}^{2 d+2(d-i)(l-1), d+(d-i)(l-1)}(-, \mathbf{Z} / l)
\end{gathered}
$$

Below we use $C_{i}$ instead of $C_{i, d}$ and $D_{i}$ instead of $D_{i, d}$ when no confusion is possible. Recall that we denote by $\tau$ the generator of $H^{0,1}(k, \mathbf{Z} / 2)$ for $\operatorname{char}(k) \neq 2$ and by $\rho$ the class of -1 in $H^{1,1}(k, \mathbf{Z} / 2)$. 
Lemma 9.1 Let $u \in \tilde{H}^{2 d, d}\left(F_{\bullet}\right), v \in \tilde{H}^{2 d^{\prime}, d^{\prime}}\left(F_{\bullet}^{\prime}\right)$. Then for l odd one has:

$$
\begin{gathered}
D_{i}(u \wedge v)=\sum_{r=0}^{i} D_{r}(u) \wedge D_{i-r}(v) \\
C_{i+1}(u \wedge v)=\sum_{r=0}^{i} C_{r+1}(u) \wedge D_{i-r}(v)+D_{r}(u) \wedge C_{i-r+1}(v)
\end{gathered}
$$

and for $l=2$ one has

$$
\begin{gathered}
D_{i}(u \wedge v)=\sum_{r=0}^{i} D_{r}(u) \wedge D_{i-r}(v)+\tau \sum_{r=0, \ldots, i-1} C_{r+1}(u) \wedge C_{i-r}(v) \\
C_{i+1}(u \wedge v)=\sum_{r=0}^{i} C_{r+1}(u) \wedge D_{i-r}(v)+D_{r}(u) \wedge C_{i-r+1}(v)+\rho C_{r+1}(u) \wedge C_{i-r+1}(v)
\end{gathered}
$$

Proof: Follows immediately from Lemma 5.7 and Theorem 6.16.

Lemma 9.2 Let $t \in \tilde{H}^{2,1}(T, \mathbf{Z} / l)$ be the tautological class. Then one has:

$$
\begin{aligned}
& C_{i+1}(u \wedge t)=C_{i}(u) \wedge t \\
& D_{i}(u \wedge t)=D_{i-1}(u) \wedge t
\end{aligned}
$$

Proof: By Lemma 5.8 we have $P(t)=\delta\left(T \rightarrow T h\left(\xi_{l} / \mathcal{O}\right)\right)\left(t_{\xi}\right)$. In view of Lemma 4.7 and the fact that $d=e\left(\xi_{l} / \mathcal{O}\right)$ we get $P(t)=t \wedge d$ i.e. $C_{i+1}(t)=0$ for all $i \geq 0, D_{1}(t)=t$ and $D_{i}(t)=0$ for $i \neq 1$. Applying Lemma 9.1 we get (9.2).

For $u \in \tilde{H}^{2 d, d}$ define;

$$
\begin{aligned}
& P^{i}(u)=D_{d-i}(u) \\
& B^{i}(u)=C_{d-i}(u)
\end{aligned}
$$

By (9.2) we have $P^{i}(u \wedge t)=P^{i}(u) \wedge t$ and $B^{i}(u \wedge t)=B^{i}(u) \wedge t$. As shown in the proof of Proposition 2.6 we can extend $P^{i}$ and $B^{i}$ to operations acting on motivic cohomology groups $\tilde{H}^{p, q}$ for all $p, q$ :

$$
P^{i}: \tilde{H}^{p, q} \rightarrow \tilde{H}^{p+2 i(l-1), q+i(l-1)}
$$




$$
B^{i}: \tilde{H}^{p, q} \rightarrow \tilde{H}^{p+2 i(l-1)+1, q+i(l-1)}
$$

For $l=2$ we denote, following the standard convention,

$$
\begin{gathered}
S q^{2 i}=P^{i} \\
S q^{2 i+1}=B^{i}
\end{gathered}
$$

Theorem 9.3 For any $i<0$ one has $P^{i}=B^{i}=0$.

Proof: This follows from Proposition 3.7.

Theorem 9.4 One has $P^{0}=I d$.

Proof: Proposition 3.8 implies that $P^{0}(u)=a u$ where $a$ is a constant. Lemma 6.17 applied to the canonical line bundle on $\mathbf{P}^{1}$ implies that $a=1$.

Lemma 9.5 One has $\beta B^{i}=0$ and $\beta P^{i}=B^{i}$.

Proof: Follows immediately from Theorem 8.4, the fact that $\beta(c)=d$ and the product formula (8.1) for the Bockstein homomorphism.

Proposition 9.6 For $u, v \in \tilde{H}^{*, *}$ and $l \neq 2$ one has:

$$
\begin{gathered}
P^{i}(u \wedge v)=\sum_{r=0}^{i} P^{r}(u) \wedge P^{i-r}(v) \\
B^{i}(u \wedge v)=\sum_{r=0}^{i}\left(B^{r}(u) \wedge P^{i-r}(v)+P^{r}(u) \wedge B^{i-r}(v)\right)
\end{gathered}
$$

For $l=2$ we get:

$$
\begin{gathered}
S q^{2 i}(u \wedge v)=\sum_{r=0}^{i} S q^{2 r}(u) \wedge S q^{2 i-2 r}(v)+\tau \sum_{s=0}^{i-1} S q^{2 s+1}(u) \wedge S q^{2 i-2 s-1}(v) \\
S q^{2 i+1}(u \wedge v)=\sum_{r=0}^{i}\left(S q^{2 r+1}(u) \wedge S q^{2 i-2 r}(v)+S q^{2 r}(u) \wedge S q^{2 i-2 r-1}(v)\right)+ \\
+\rho \sum_{s=0}^{i-1} S q^{2 s+1}(u) \wedge S q^{2 i-2 s-1}(v)
\end{gathered}
$$


Proof: Follows immediately from Lemma 9.1 and the vanishing result 9.3.

Lemma 9.7 For $u \in \tilde{H}^{2 n, n}$ one has $P^{n}(u)=u^{l}$.

Proof: Follows from Lemma 5.10.

Lemma 9.8 For $u \in \tilde{H}^{p, q}$ and $n>p-q, n \geq q$ one has $P^{n}(u)=0$.

Proof: Let $i=n+q-p$ and $j=n-q$. Then $\sigma_{s}^{i} \sigma_{t}^{j}(u)$ is in $\tilde{H}^{2 n, n}$. By Lemma 9.7 we get

$$
\sigma_{s}^{i} \sigma_{t}^{j} P^{n}(u)=\left(\sigma_{s}^{i} \sigma_{t}^{j}(u)\right)^{l}
$$

By our assumption $i>0$ and the right hand side is zero because the diagonal map $S_{s}^{1} \rightarrow S_{s}^{1} \wedge S_{s}^{1}$ is zero in $H_{\bullet}$.

We will also use the total power operation:

$$
R: \tilde{H}^{*, *} \rightarrow \tilde{H}^{*, *}\left[\left[c, d, d^{-1}\right]\right] /\left(c^{2}=\tau d+\rho c\right)
$$

where $\tau=\rho=0$ for $l \neq 2$ and for $l=2, \tau$ and $\rho$ are as in Theorem 6.16. We define $R$ by the formula

$$
R(u)=\sum_{i}\left(B^{i-1}(u) c d^{-i}+P^{i}(u) d^{-i}\right)
$$

For $l=2$ this becomes

$$
R(u)=\sum_{i}\left(S q^{2 i-1}(u) c d^{-i}+S q^{2 i}(u) d^{-i}\right)
$$

For $u \in \tilde{H}^{2 n, n}$ we have $d^{2 n} R(u)=P(u)$. Together with Lemma 5.7 this implies that for any $u$ and $v$ one has

$$
R(u v)=R(u) R(v)
$$

where the right hand side is to be computed in the ring $H^{*, *}\left[\left[c, d, d^{-1}\right]\right] /\left(c^{2}=\right.$ $\tau d+\rho c)$. 


\section{Adem relations}

Lemma 10.1 Let $d_{1}, c_{1}$ be generators of $H^{*, *}\left(B S_{l}, \mathbf{Z} / l\right)$ and $c_{2}, d_{2}$ the generators of the ring appearing in the definition of $R(u)$. Then one has:

$$
\begin{gathered}
R\left(d_{1}\right)=d_{1}\left(1-d_{1} / d_{2}\right)^{l-1} \\
R\left(c_{1}\right)=\left(c_{1}+\left(d_{1} / d_{2}\right) c_{2}\right)\left(1-d_{1} / d_{2}\right)^{l-2}
\end{gathered}
$$

Proof: By transfer argument we may assume that $k$ contains a primitive l-th root of unity $\zeta$. Consider the map

$$
\phi=p_{\zeta}^{*}: H^{*, *}\left(B S_{l}\right) \rightarrow H^{*, *}\left(B \mu_{l}\right)
$$

defined by $\zeta$. Then $\phi$ is a mono (by Lemma 6.12) and by Lemmas $6.13,6.15$ one has

$$
\begin{gathered}
\phi\left(d_{1}\right)=-u^{l-1} \\
\phi\left(c_{1}\right)=-v u^{l-2}
\end{gathered}
$$

where $u$ and $v$ are the generators from Theorem 6.10. We get:

$$
R\left(u^{l-1}\right)=R(u)^{l-1}=d_{2}^{1-l} P(u)^{l-1}=d_{2}^{1-l}\left(u^{l}+u d_{2}\right)^{l-1}
$$

where the last equality holds by Lemma 6.17 . The right hand side equals to $-\phi\left(d_{1}\right)\left(1-\phi\left(d_{1}\right) / d_{2}\right)^{l-1}$ which implies (10.1). For $R\left(v u^{l-2}\right)$ we get

$$
\begin{gathered}
R\left(v u^{l-2}\right)=R(v) R(u)^{l-2}=d_{2}^{2-l} R(v) P(u)= \\
=d_{2}^{2-l}\left(P^{1}(v)+B^{0}(v) c_{2}+P^{0}(v) d_{2}\right)\left(u^{l}+u d_{2}\right)^{l-2}
\end{gathered}
$$

By Theorem 9.4 $P^{0}(v)=v$, by Lemma 9.5 and since $\beta(v)=u, B^{0}(v)=u$. By Lemma 9.8, $P^{1}(v)=0$ and therefore our expression equals

$d_{2}^{1-l}\left(u^{l-1} c_{2}+v u^{l-2} d_{2}\right)\left(u^{l-2}+d_{2}\right)^{l-2}=d_{2}^{1-l}\left(-\phi\left(d_{1}\right) c_{2}-\phi\left(c_{1}\right) d_{2}\right)\left(-\phi\left(d_{1}\right)+d_{2}\right)^{l-2}$

This implies (10.2).

Theorem 10.2 Let $l=2$ and $0<a<2 b$. Then for $a+b=0(\bmod 2)$ one has:

$$
S q^{a} S q^{b}= \begin{cases}\sum_{j=0}^{[a / 2]}\left(\begin{array}{c}
b-1-j \\
a-2 j
\end{array}\right) S q^{a+b-j} S q^{j} & \text { for } a, b \text { odd } \\
\sum_{j=0}^{[a / 2]} \tau^{j \bmod 2}\left(\begin{array}{c}
b-1-j \\
a-2 j
\end{array}\right) S q^{a+b-j} S q^{j} & \text { for } a, b \text { even }\end{cases}
$$


and for $a+b=1(\bmod 2)$ one has:

$$
S q^{a} S q^{b}=\sum_{j=0}^{[a / 2]}\left(\begin{array}{c}
b-1-j \\
a-2 j
\end{array}\right) S q^{a+b-j} S q^{j}+\rho^{(j+1) \bmod 2} S(a, b)
$$

where:

$$
S(a, b)= \begin{cases}\left(\begin{array}{c}
b-1-j \\
a-2 j
\end{array}\right) S q^{a+b+j} S q^{j-1} & \text { for } a \text { even, } b \text { odd } \\
\left(\begin{array}{c}
b-1-j \\
a-1-2 j
\end{array}\right) S q^{a+b+j-1} S q^{j} & \text { for } a \text { odd, } b \text { even }\end{cases}
$$

Proof: Consider the class $P(P(u))$ for $u \in \tilde{H}^{2 n, n}$. Denote by $d_{1}, c_{1}$ the generators of the cohomology of $B S_{l}$ appearing when the first $P$ is applied and by $d_{2}, c_{2}$ the generators of the cohomology of $B S_{l}$ appearing when the second $P$ is applied. According to the symmetry theorem 7.7 the resulting expression is symmetric with respect to the exchange of $\left(d_{1}, c_{1}\right)$ and $\left(d_{2}, c_{2}\right)$. We have (to simplify the notations we sometimes omit $u$ from our expressions):

$$
\begin{gathered}
P(u)=\sum_{i} S q^{2 n-2 i-1} c d^{i}+S q^{2 n-2 i} d^{i} \\
P(P(u))=d_{2}^{2 n} R(P(u))=d_{2}^{2 n} \sum_{i}\left(R\left(S q^{2 n-2 i-1}\right) R\left(c_{1}\right)+R\left(S q^{2 n-2 i}\right)\right) R\left(d_{1}\right)^{i}= \\
=\sum_{i, j} d_{1}^{i}\left(d_{1}+d_{2}\right)^{i} d_{2}^{2 n-j-i}\left(\left(S q^{2 j-1} S q^{2 n-2 i-1} c_{2}+S q^{2 j} S q^{2 n-2 i-1}\right)\left(c_{1}+\left(d_{1} / d_{2}\right) c_{2}\right)+\right. \\
\left.+S q^{2 j-1} S q^{2 n-2 i} c_{2}+S q^{2 j} S q^{2 n-2 i}\right)
\end{gathered}
$$

Consider the coefficients in this expression at $1, c_{1}, c_{2}$ and $c_{1} c_{2}$. At 1 we have:

$$
\sum_{i, j} d_{1}^{i}\left(d_{1}+d_{2}\right)^{i} d_{2}^{2 n-j-i}\left(S q^{2 j} S q^{2 n-2 i}+\tau d_{1} S q^{2 j-1} S q^{2 n-2 i-1}\right)
$$

At $c_{1} c_{2}$ we have:

$$
\sum_{i, j} d_{1}^{i}\left(d_{1}+d_{2}\right)^{i} d_{2}^{2 n-j-i} S q^{2 j-1} S q^{2 n-2 i-1}
$$


At $c_{1}$ we have:

$$
\sum_{i, j} d_{1}^{i}\left(d_{1}+d_{2}\right)^{i} d_{2}^{2 n-j-i} S q^{2 j} S q^{2 n-2 i-1}
$$

At $c_{2}$ we have:

$$
\begin{gathered}
\sum_{i, j} d_{1}^{i}\left(d_{1}+d_{2}\right)^{i} d_{2}^{2 n-j-i} S q^{2 j-1} S q^{2 n-2 i}+ \\
+\sum_{i, j} d_{1}^{i+1}\left(d_{1}+d_{2}\right)^{i} d_{2}^{2 n-j-i-1}\left(\rho S q^{2 j-1} S q^{2 n-2 i-1}+S q^{2 j} S q^{2 n-2 i-1}\right)
\end{gathered}
$$

Set $p=i+r, q=2 n-j-r$. Then coefficient at $d_{1}^{p} d_{2}^{q}$ is

$$
\sum_{i}\left(\begin{array}{c}
i \\
p-i
\end{array}\right) S q^{4 n-2 p-2 q+2 i} S q^{2 n-2 i}+\tau\left(\begin{array}{c}
i-1 \\
p-i
\end{array}\right) S q^{4 n-2 p-2 q+2 i-1} S q^{2 n-2 i+1}
$$

Coefficient at $c_{1} c_{2} d_{1}^{p} d_{2}^{q}$ is

$$
\sum_{i}\left(\begin{array}{c}
i \\
p-i
\end{array}\right) S q^{4 n-2 p-2 q+2 i-1} S q^{2 n-2 i-1}
$$

Coefficient at $c_{1} d_{1}^{p} d_{2}^{q}$ is

$$
\sum_{i}\left(\begin{array}{c}
i \\
p-i
\end{array}\right) S q^{4 n-2 p-2 q+2 i} S q^{2 n-2 i-1}
$$

Coefficient at $c_{2} d_{1}^{p} d_{2}^{q}$ is

$$
\begin{aligned}
\sum_{i}\left(\begin{array}{c}
i \\
p-i
\end{array}\right) & S q^{4 n-2 p-2 q+2 i-1} S q^{2 n-2 i}+\left(\begin{array}{c}
i \\
p-i-1
\end{array}\right) S q^{4 n-2 p-2 q+2 i} S q^{2 n-2 i-1} \\
& +\rho \sum_{i}\left(\begin{array}{c}
i \\
p-i-1
\end{array}\right) S q^{4 n-2 p-2 q+2 i-1} S q^{2 n-2 i-1}
\end{aligned}
$$

Consider the coefficient at $c_{1} c_{2} d_{1}^{p} d_{2}^{q}$ where $p=2^{s}-1$ for sufficiently large $s$ and $q=x$. For $p$ of this form, the coefficient $\left(\begin{array}{c}i \\ p-i\end{array}\right)$ is non-zero if and only if $i=p$ (follows from [7, ]) and we conclude that our coefficient is $S q^{4 n-2 x-1} S q^{2 n-2^{s+1}+1}$. By symmetry it equals to the coefficient at $c_{1} c_{2} d_{1}^{q} d_{2}^{p}$. 
Setting $a=4 n-2 x-1, b=2 n-2^{s+1}+1$ and $j=2 n-2 i-1$ and using the fact that

$$
\left(\begin{array}{l}
u \\
v
\end{array}\right)=\left(\begin{array}{l}
2 u \\
2 v
\end{array}\right) \bmod 2
$$

we can write the later as

$$
\sum_{j=1 \bmod 2}\left(\begin{array}{c}
2 n-1-j \\
a-2 j-1
\end{array}\right) S q^{a+b-j} S q^{j}
$$

From the standard relation

$$
\left(\begin{array}{c}
u \\
v-1
\end{array}\right)=\left(\begin{array}{c}
u+1 \\
v
\end{array}\right)+\left(\begin{array}{l}
u \\
v
\end{array}\right)
$$

and the fact that

$$
\left(\begin{array}{l}
u \\
v
\end{array}\right)=0 \bmod 2
$$

if $u$ is even and $v$ is odd we get the first of the equalities stated in the theorem. A very similar argument starting with the equality between the coefficients at $c_{1} d_{1}^{p} d_{2}^{q}$ and $c_{2} d_{1}^{q} d_{2}^{p}$ gives the third equality - the case of even $a$ and odd $b$. To prove the case when both $a$ and $b$ are even consider the coefficient at $d_{1}^{p} d_{2}^{q}$. Consider the second part of this coefficient i.e. the sum

$$
\begin{aligned}
& \sum_{i}\left(\begin{array}{c}
i-1 \\
p-i
\end{array}\right) S q^{4 n-2 p-2 q+2 i-1} S q^{2 n-2 i+1}= \\
= & \sum_{j=i-1}\left(\begin{array}{c}
j \\
p-1-j
\end{array}\right) S q^{4 n-2(p-1)-2 q+2 j-1} S q^{2 n-2 j-1}
\end{aligned}
$$

This is the coefficient at $c_{1} c_{2} d_{1}^{p-1} d_{2}^{q}$ which is equal to the coefficient at $c_{1} c_{2} d_{1}^{q} d_{2}^{p-1}$ i.e. to

$$
\begin{aligned}
& \sum_{j=i-1}\left(\begin{array}{c}
j \\
q-j
\end{array}\right) S q^{4 n-2(p-1)-2 q+2 j-1} S q^{2 n-2 j-1}= \\
& =\sum_{i}\left(\begin{array}{c}
i-1 \\
q-i+1
\end{array}\right) S q^{4 n-2 p-2 q+2 i-1} S q^{2 n-2 i+1}
\end{aligned}
$$


Equating our new expression for the coefficient at $d_{1}^{p} d_{2}^{q}$ with the old expression for the coefficient at $d_{1}^{q} d_{2}^{p}$ we get

$$
\begin{gathered}
\sum_{i}\left(\begin{array}{c}
i \\
p-i
\end{array}\right) S q^{4 n-2 p-2 q+2 i} S q^{2 n-2 i}= \\
=\sum_{i}\left(\begin{array}{c}
i \\
q-i
\end{array}\right) S q^{4 n-2 p-2 q+2 i} S q^{2 n-2 i}+ \\
+\tau \sum_{i}\left(\left(\begin{array}{c}
i-1 \\
q-i+1
\end{array}\right)+\left(\begin{array}{c}
i-1 \\
q-i
\end{array}\right)\right) S q^{4 n-2 p-2 q+2 i-1} S q^{2 n-2 i+1}
\end{gathered}
$$

Setting again $p=2^{s}-1, a=2 n-2 q$ and $b=2 n-2 p$ and using the standard relations between the binomial coefficients one recovers the identity for $S q^{a} S q^{b}$ when both $a$ and $b$ are even. Finally, to get the identity in the case when $a$ is odd and $b$ is even one uses Lemma 9.5, the identity for $a$ and $b$ even and the fact that $\beta(\tau)=\rho$.

The proof of the following theorem which provides Adem relations for odd $l$ follows the same line of arguments as the proof of the corresponding topological fact given in $[7$,$] .$

Theorem 10.3 For $l>2$ and $0<a<l b$ one has:

$$
P^{a} P^{b}=\sum_{t=0}^{[a / l]}(-1)^{a+t}\left(\begin{array}{c}
(l-1)(b-t)-1 \\
a-l t
\end{array}\right) P^{a+b-t} P^{t}
$$

And for $0 \geq a \geq l b$ one has:

$$
\begin{gathered}
P^{a} B^{b}=\sum_{t=0}^{[a / l]}(-1)^{a+t}\left(\begin{array}{c}
(l-1)(b-t) \\
a-l t
\end{array}\right) B^{a+b-t} P^{t}+ \\
+\sum_{t=0}^{[(a-1) / l]}(-1)^{a+t-1}\left(\begin{array}{c}
(l-1)(b-t)-1 \\
a-l t-1
\end{array}\right) P^{a+b-t} B^{t}+
\end{gathered}
$$

Proof: These relations are exactly the same as the Adem relations in the topological Steenrod algebra for odd coefficients. The proof of these relations given in [7, Theorem VIII.1.6] works in exactly the same way in the motivic context as in the topological one if one replaces the reference to [7, Corollary VIII.1.2] with the reference to our symmetry theorem 7.7. The apparent difference in signs between our situation and the situation of [7] is explained by the fact that the image of $d$ in the cohomology of $B \mathbf{Z} / l$ is $-u^{l-1}=-w_{2 l-2}$. 


\section{Motivic Steenrod algebra}

Define the motivic Steenrod algebra $A^{*, *}(k, \mathbf{Z} / l)$ as the subalgebra in the algebra of bistable cohomological operations in the motivic cohomology with $\mathbf{Z} / l$ coefficients over $k$ generated by operations $P^{i}, B^{i}, i \geq 0$ and operations of the form $u \mapsto a u$ where $a \in H^{*, *}(k, \mathbf{Z} / l)$.

Let $I=\left(\epsilon_{0}, s_{1}, \epsilon_{1}, s_{2}, \ldots, s_{k}, \epsilon_{k}\right)$ be a sequence where $\epsilon_{i} \in\{0,1\}$ and $s_{i}$ are non-negative integers. Denote by $P^{I}$ the product

$$
P^{I}=\beta^{\epsilon_{0}} P^{s_{1}} \ldots P^{s_{k}} \beta^{\epsilon_{k}}
$$

A sequence $I$ is called admissible if $s_{i} \geq l s_{i+1}+\epsilon_{i}$. Monomials $P^{I}$ corresponding to admissible sequences are called admissible monomials.

Lemma 11.1 Admissible monomials generate $A^{*, *}(k, \mathbf{Z} / l)$ as a left $H^{*, *}$ module.

Proof: This follows from the Adem relations and the Cartan formula 9.6.

Our next goal is to show that the admissible monomials are linearly independent with respect to the left $H^{*, *}$-module structure on $A^{*, *}$ and, therefore, form a basis of this module. Consider the submodule $H^{*,>0} A^{*, *}$ in $A^{*, *}$. The Cartan formulas 9.6 imply that its is a two-sided ideal in $A^{*, *}$. Set

$$
A_{\text {rig }}^{*, *}=A^{*, *} / H^{*,>0} A^{*, *}
$$

Using again the Cartan formula one observes that the action of $A^{*, *}$ on $H^{*, *}(X)$ defines an action of $A_{r i g}^{*, *}$ on $H^{*, *}(X) / H^{*,>0} H^{*, *}(X)$. Theorem 6.10 immediately implies the following result.

Lemma 11.2 For any $l$ and any $k$ one has:

$$
H^{*, *}\left(B \mu_{l}\right) / H^{*,>0} H^{*, *}\left(B \mu_{l}\right)=\mathbf{Z} / l[u, v] /\left(v^{2}=0\right)
$$

Lemma 10.1 implies the following.

Lemma 11.3 Let $u$ and $v$ be as in Lemma 11.2. Then one has the following equalities in $H^{*, *}\left(B \mu_{l}\right) / H^{*,>0} H^{*, *}\left(B \mu_{l}\right)$ :

$$
\begin{array}{ll}
\beta(v)=u & P^{i}(v)=0 \text { for } i>0 \\
\beta\left(u^{k}\right)=0 & P^{i}\left(u^{k}\right)=\left(\begin{array}{c}
k \\
i
\end{array}\right) u^{k+i(l-1)}
\end{array}
$$


Let $d(I)$ be the degree of an admissible monomial $P^{I}$ i.e. the integer such that $P^{I}$ is an operation from $\tilde{H}^{*, *}$ to $\tilde{H}^{*+d(I), *+q}$.

Proposition 11.4 For any $n \geq 0$ there exists $N$ and an element $w$ in $H^{*, *}\left(\left(B \mu_{L}\right)^{N}\right)$ such that the elements $P^{I}(w)$, for all $I$ such that $d(I) \leq n$, are linearly independent with respect to the left $H^{*, *}$-module structure on $H^{*, *}\left(\left(B \mu_{L}\right)^{N}\right)$.

Proof: It is sufficient to show that there exists $w$ such that $P^{I}(w)$ are linerly independent in $H^{*, *}\left(\left(B \mu_{L}\right)^{N}\right) / H^{*,>0} H^{*, *}\left(\left(B \mu_{L}\right)^{N}\right)$ with respect to the $\mathbf{Z} / l$ module structure. One starts with Lemma 11.3 and uses exactly the same reasoning as in the proof of [7, Proposition VI.2.4]

The following is an immediate corollary of the proof of Proposition 11.5.

Corollary 11.5 The admissible monomials are linearly independent with respect to the left $H^{*, *}$-module structure on $A^{*, *}$.

Let $l$ be an odd prime. Denote by $A_{\text {top }}^{*, *}$ the $\mathbf{Z} / l$-submodule of $A^{*, *}$ generated by the admissible monomials. The Adem relations (Theorem 10.3) show that $A_{t o p}^{*, *}$ is a subring of $A^{*, *}$. Together with Corollary 11.5 they imply that $A_{\text {top }}^{*, *}$ is isomorphic to the usual topological Steenrod algebra with the second grading given by assigning the weight $(l-1) i$ to $P^{i}$ and the weight 0 to $\beta$. The Cartan formula (Proposition 9.6) shows that the action of elements of $A_{\text {top }}^{*, *}$ on products of motivic cohomology classes has the same expansion as in topology. Taken together these observations show that all the standard results about the topological Steenrod algebra and its dual in the case of odd coefficients translate without change to the motivic context. In what follows we consider both the odd and even coefficients cases but give the proofs only in the even case where the motivic Steenrod algebra has a more complicated structure than its topological counterpart.

Below we denote by $A^{*, *} \otimes_{H^{*, *}} A^{*, *}$ the tensor product of left $H^{*, *}$-modules $A^{*, *}$. The action of $A^{*, *}$ on $\tilde{H}^{*, *}(X)$ is not, in general, $H^{*, *}$-linear. Hence, we can not speak of the action of $A^{*, *} \otimes_{H^{*, *}} A^{*, *}$ on $\tilde{H}^{*, *}(X) \otimes_{H^{*, *}} \tilde{H}^{*, *}(Y)$. However, since the action of $A^{*, *}$ is $\mathbf{Z} / l$-linear it defines an action of $A^{*, *} \otimes \mathbf{z} / l$ $A^{*, *}$ on $\tilde{H}^{*, *}(X) \otimes_{\mathbf{z} / l} \tilde{H}^{*, *}(Y)$. If $x, y$ are two elements of $A^{*, *} \otimes_{\mathbf{z} / l} A^{*, *}$ which become equal in the tensor product over $H^{*, *}$ then for any $u$ in $\tilde{H}^{*, *}(X) \otimes \mathbf{z} / l$ $\tilde{H}^{*, *}(Y)$ we have $x(u)=x(v)$ in $\tilde{H}^{*, *}(X) \otimes_{H^{*, *}} \tilde{H}^{*, *}(Y)$. Therefore, for $x$ in $A^{*, *} \otimes_{\mathbf{z} / l} A^{*, *}$ and $u$ in $\tilde{H}^{*, *}(X) \otimes_{\mathbf{z} / l} \tilde{H}^{*, *}(Y)$ there is a well defined element $x(u)$ in $\tilde{H}^{*, *}(X) \otimes_{H^{*, *}} \tilde{H}^{*, *}(Y)$. 
Lemma 11.6 For any element $x$ of $A^{*, *}$ there exists a unique element

$$
\psi^{*}(x)=\sum x_{i}^{\prime} \otimes x_{i}^{\prime \prime}
$$

of $A^{*, *} \otimes_{H^{*, *}} A^{*, *}$ such that for any $X$ and any $u \in \tilde{H}^{p, *}(X), v \in \tilde{H}^{*, *}(X)$ one has

$$
x(u v)=\sum(-1)^{\operatorname{dim}\left(x_{i}^{\prime \prime}\right) p} x_{i}^{\prime}(u) x_{i}^{\prime \prime}(v)
$$

Proof: Exactly parallel to the proof of [5, Lemma 1, p.154] where Proposition 11.4 is used to prove uniqueness.

We will need the following lemma below.

Lemma 11.7 Let $x$ be an element of $A^{*, *}$ and $\psi^{*}(x)=\sum x_{i}^{\prime} \otimes x_{i}^{\prime \prime}$. Then for $a \in H^{* * *}$ one has:

$$
\psi^{*}(x a)=\sum x_{i}^{\prime} a \otimes x_{i}^{\prime \prime}=\sum x_{i}^{\prime} \otimes x_{i}^{\prime \prime} a
$$

Proof: By uniqueness part of Lemma 11.6 it is enough to check that for any $u, v \in \tilde{H}^{*, *}(X)$ one has:

$$
\left(\sum x_{i}^{\prime} a \otimes x_{i}^{\prime \prime}\right)(u \otimes v)=\left(\sum x_{i}^{\prime} \otimes x_{i}^{\prime \prime} a\right)(u \otimes v)=(x a)(u v)
$$

This follows immediately from definitions.

Since $H^{*, *}$ is not in the center of $A^{*, *}$, the ring structure on $A^{*, *} \otimes_{\mathbf{z} / l} A^{*, *}$ does not define a ring structure on $A^{*, *} \otimes_{H^{*, *}} A^{*, *}$. The best we can get in general is an action of $A^{*, *} \otimes_{H^{*, *}} A^{*, *}$ on $A^{*, *} \otimes_{\mathbf{Z} / l} A^{*, *}$ with values in $A^{*, *} \otimes_{H^{*, *}} A^{*, *}$ given by

$$
(u \otimes v)\left(u^{\prime} \otimes v^{\prime}\right)=u u^{\prime} \otimes v v^{\prime}
$$

We say that an element $f$ of $A^{*, *} \otimes_{H^{* * *}} A^{*, *}$ is an operator-like element if for any two elements $x, y$ of $A^{*, *} \otimes \mathbf{z} / l A^{*, *}$ which belome equal in the tensor product over $H^{*, *}$ one has $f x=f y$. For an operator-line element $f$ and any other element $x$ the product $f x$ is well defined. If $f$ and $g$ are two operator-like elements the product $f g$ is again operator-line. This shows that operator-like elements form a ring which we denote by $\left(A^{*, *} \otimes_{H^{*, *}} A^{*, *}\right)_{r}$.

Lemma 11.8 For any $x$ in $A^{*, *}, \psi^{*}(x)$ is an operator-like element. The map $\psi^{*}: A^{*, *} \rightarrow\left(A^{*, *} \otimes_{H^{* * *}} A^{*, *}\right)_{r}$ is a ring homomorphism. 
Proof: Let $y, z$ be two elements of $A^{*, *} \otimes_{\mathbf{z} / l} A^{*, *}$ which become equal modulo $H^{*, *}$. To check that $\psi^{*}(x) y=\psi^{*}(x) z$ it is sufficient, in view of Proposition 11.4 , to check that for any $X$ and any $w_{1}, w_{2} \in \tilde{H}^{* * *}(X)$ one has $\psi^{*}(x) y\left(w_{1} \otimes\right.$ $\left.w_{2}\right)=\psi^{*}(x) z\left(w_{1} \otimes w_{2}\right)$. Let $c: \tilde{H}^{*, *}(X) \otimes \tilde{H}^{*, *}(X) \rightarrow \tilde{H}^{*, *}(X)$ be the cup product. Then by definition of $\psi$ we have

$$
\begin{aligned}
& \psi^{*}(x) y\left(w_{1} \otimes w_{2}\right)=x\left(c\left(y\left(w_{1} \otimes w_{2}\right)\right)\right) \\
& \psi^{*}(x) z\left(w_{1} \otimes w_{2}\right)=x\left(c\left(z\left(w_{1} \otimes w_{2}\right)\right)\right)
\end{aligned}
$$

Our assumption on $y, z$ implies that $c\left(y\left(w_{1} \otimes w_{2}\right)\right)=c\left(z\left(w_{1} \otimes w_{2}\right)\right)$.

To prove that $\psi^{*}$ is a ring homomorphism we have to check that for $x, y \in A^{*, *}$ and $w_{1}, w_{2} \in \tilde{H}^{*, *}(X)$ we have

$$
\psi(x y)\left(w_{1} \otimes w_{2}\right)=\psi(x)\left(\psi(y)\left(w_{1} \otimes w_{2}\right)\right)
$$

This follows immediately from definitions.

Lemma 11.9 The comultiplication map $\psi^{*}$ is associative and commutative.

Proof: The associativity follows immediately from the definition. To verify commutativity it is sufficient, in view of Lemma 11.8, to verify it on generators of the algebra $A^{*, *}$ i.e. on operations $P^{i}$ and $B^{i}$. For this operations commutativity follows directly from the Cartan formulas (Proposition 9.6).

\section{Structure of the dual to the motivic Steenrod alge- bra}

Let $f: A^{*, *} \rightarrow H^{*, *}$ be a homomorphism of left $H^{*, *}$-modules. Such a homomorphism is said to be homogeneous of bidegree $(p, q)$ if for any $i, j \geq 0$ it takes $A^{i, j}$ to $H^{i-p, j-q}$. We denote by $A_{*, *}$ the "bigraded dual" to $A^{*, *}$ i.e. the group of the left $H^{*, *}$-module maps from $A^{*, *}$ to $H^{*, *}$ which are finite sums of homogeneous maps.

Let $P^{I}$ be the basis of admissible monomials in $A^{*, *}$ and $\theta(I)^{*}$ the dual basis in $A_{*, *}$. An element $x$ from $A_{p, q}$ can be written uniquely as a sum of the form

$$
x=\sum a_{I} \theta(I)^{*}
$$

where $\theta(I)^{*} \in A^{r, s}$ and $a_{I} \in H^{i, j}$ such that $p=r-i, q=s-j$. Since $H^{i, j}=0$ for $i>j$ we get

$$
r-s=p-q+i-j \leq p-q
$$


Since for any $I, r \geq 2 s$ and $s \geq 0$ we further have

$$
2 s \leq r \leq p-q+s
$$

which implies:

$$
0 \leq s \leq p-q ; \quad 2 s \leq r \leq 2(p-q)
$$

Therefore, the sum (12.1) is always finite. This fact implies in particular that taking the bigraded dual to $A_{*, *}$ we get back the original $A^{*, *}$. It also implies that $A_{*, *}$ is a free $H^{*, *}$ module.

We have a homomorphism $H^{*, *} \rightarrow A_{*, *}$ which takes $a \in H^{p, q}$ to the map $\phi \mapsto a \phi(1)$ which lies in $A_{-p,-q}$ and which we also denote by $a$. The homomorphism $\psi^{*}$ defines a homomorphism $\phi_{*}: A_{*, *} \otimes A_{*, *} \rightarrow A_{*, *}$. Lemma 11.9 immediately implies the following result.

Proposition 12.1 The homomorphism $\phi_{*}$ makes $A_{*, *}$ into an associative ring which is graded commutative with respect to the first grading.

Let $e^{i} \in A^{p_{i}, q_{i}}$ be a basis of $A^{*, *}$ over $H^{*, *}$ such that:

1. $e_{0}=1$ and $q_{i}>0$ for $i>0$

2. $p_{i} \geq 2 q_{i}$

3. for any $q$ there are only finitely many $i$ with $q_{i} \leq q$.

An example of such a basis is given by the basis of admissible monomials. Let $e_{i}$ be the dual basis of $A_{*, *}$. Let $X$ be a smooth scheme over $k$. Then $H^{p, q}(X)=0$ for $p>q+\operatorname{dim}(X)$ and therefore for any $w \in H^{*, *}(X)$ there is only finitely many $i$ 's such that $e^{i}(w) \neq 0$. We can define a map

$$
\lambda^{*, *}: H^{*, *}(X) \rightarrow H^{*, *}(X) \otimes_{H^{*, *}} A_{*, *}
$$

by the formula

$$
\lambda^{*, *}(w)=\sum e^{i}(w) \otimes e_{i}
$$

The following lemma is straightforward.

Lemma 12.2 The map $\lambda^{*, *}$ is a ring homomorphism which does not depend on the choice of $e^{i}$. 
Note that in the case when $X=\operatorname{Spec}(k)$ this homomorphism does not coincide with the "scalar" map $H^{*, *} \rightarrow \mathcal{A}_{*, *}$ which is described above since the action of $e_{i}$ 's on $H^{*, *}$ may be nontrivial. In particular $\lambda^{*, *}$ is not a $H^{*, *}$ module homomorphism.

If we consider $B \mu_{l}$ as a colimit of smooth schemes we can write a formal analog of $\lambda^{* * *}$. In particular for the canonical generators $u$ and $v$ we get:

$$
\begin{aligned}
& \lambda^{*, *}(v)=\sum_{i=0}^{\infty}\left(v u^{i} \otimes x_{i}+u^{i} \otimes y_{i}\right) \\
& \lambda^{*, *}(u)=\sum_{i=0}^{\infty}\left(v u^{i} \otimes x_{i}^{\prime}+u^{i} \otimes y_{i}^{\prime}\right)
\end{aligned}
$$

where $x_{i}, y_{i}, x_{i}^{\prime}, y_{i}^{\prime}$ are some well defined elements in $\mathcal{A}_{*, *}(k, \mathbf{Z} / l)$. We denote:

$$
\begin{gathered}
\xi_{i}=y_{l^{i}}^{\prime} \in \mathcal{A}_{2\left(l^{i}-1\right), l^{i}-1}(k, \mathbf{Z} / l) \\
\tau_{i}=y_{l^{i}} \in \mathcal{A}_{2 l^{i}-1, l^{i}-1}(k, \mathbf{Z} / l)
\end{gathered}
$$

Since for any basis of $A^{*, *}$ such that $e^{0}=1$ we have $\operatorname{dim}\left(e^{i}\right)>0$ for $i \neq 0$ we conclude that $\xi_{0}=1$. Denote by $M_{k}$ the monomial $P^{l^{k-1}} P^{k^{k-2}} \ldots P^{1}$.

Lemma 12.3 We have in $H^{*, *}\left(B \mu_{l}\right)$ :

$$
M_{k}(u)=u^{l^{k}} \quad M_{k} \beta(v)=u^{l^{k}}
$$

If $f$ is any monomial in $P^{i}$ and $\beta$ other than $M_{k}$ (resp. other than $M_{k} \beta$ ) then $f(u)=0$ (resp. $f(v)=0)$.

Proof: The equations (12.2) follow from Lemma 9.7 and the fact that $\beta(v)=u$. The other statement follows from Lemma 9.8, Lemma 6.17 and multiplicativity of $P$.

Taking the basis of admissible monomials to compute $\lambda^{*, *}$ and using Lemma 12.3 we conclude that

$$
\begin{aligned}
& \lambda^{*, *}(v)=v \otimes 1+\sum_{i=0}^{\infty} u^{l^{i}} \otimes \tau_{i} \\
& \lambda^{*, *}(u)=u \otimes 1+\sum_{i=0}^{\infty} u^{l^{i}} \otimes \xi_{i} .
\end{aligned}
$$


For an element $\phi$ in $A^{*, *}$ and an element $\psi$ in $A_{*, *}$ let $\langle\psi, \phi\rangle \in H^{*, *}$ be the value of $\psi$ on $\phi$. Then we have:

$$
\begin{gathered}
\phi(u)=\left\langle\xi_{0}, \phi\right\rangle u+\sum_{i}\left\langle\tau_{i}, \phi\right\rangle v^{l^{i}} \\
\phi(v)=\sum_{i}\left\langle\xi_{i}, \phi\right\rangle v^{l^{i}}
\end{gathered}
$$

Let $I$ be a sequence of the form $\left(\epsilon_{0}, r_{1}, \epsilon_{1}, r_{2}, \ldots,\right)$ where $\epsilon_{i} \in\{0,1\}, r_{i} \geq 0$ are nonnegative integers and $I$ has only finitely many nontrivial terms. Following [5] we set:

$$
\omega(I)=\tau_{0}^{\epsilon_{0}} \xi_{1}^{r_{1}} \tau_{1}^{\epsilon_{1}} \xi_{2}^{r_{2}} \ldots
$$

in $\mathcal{A}_{p(I), q(I)}(k, \mathbf{Z} / l)$ where

$$
\begin{gathered}
p(I)=\epsilon_{0}+\sum_{i \geq 1}\left(\epsilon_{i}\left(2 l^{i}-1\right)+2 r_{i}\left(l^{i}-1\right)\right) \\
q(I)=\sum_{i \geq 1}\left(\epsilon_{i}+r_{i}\right)\left(l^{i}-1\right) .
\end{gathered}
$$

and

$$
\theta(I)=\beta^{\epsilon_{0}} P^{s_{1}} \beta^{\epsilon_{1}} P^{s_{2}} \ldots
$$

where

$$
s_{n}=\sum_{i \geq n}\left(\epsilon_{i}+r_{i}\right) l^{i-n}
$$

Simple computation shows that $\theta(I)$ belongs to $\mathcal{A}^{p(I), q(I)}(k, \mathbf{Z} / l)$.

In the following theorem we consider, following [4], the lexicographical order on the set of sequences $I$ such that $(1,2,0, \ldots)<(0,0,1, \ldots)$.

Theorem 12.4 Then, for $I<J$ one has $\langle\theta(I), \omega(J)\rangle=0$ and for $I=J$ one has $\langle\theta(I), \omega(J)\rangle= \pm 1$

Proof: The value of $\langle\theta(I), \omega(J)\rangle$ is a homogeneous element of $H^{*, *}$ of degree zero. Hence, it is sufficient to show that the image of $\langle\theta(I), \omega(J)\rangle$ in $H^{*, *} / H^{*,>0}$ is 0 or \pm 1 depending on whether $I<J$ or $I=J$. This is done using the action of $A^{*, *} / A^{*,>0}$ on $H^{*, *}\left(B \mu_{l}\right) / H^{*,>0} H^{*, *}\left(B \mu_{l}\right)$ described in Lemma 11.3 in exactly the same way as in the proof of $[5$, Lemma 8, p.160]. 
Corollary 12.5 Elements $\omega(I)$ (resp. $\theta(I))$ form a basis of the $H^{*, *}$-module $\mathcal{A}_{*, *}(k, \mathbf{Z} / l)\left(\operatorname{resp} . \mathcal{A}^{*, *}(k, \mathbf{Z} / l)\right)$.

Proof: Elements $\theta(I)$ are exactly the admissible monomials. They form a basis of $A^{*, *}$ by Lemma 11.1 and Corollary 11.5. The fact that elements $\omega(I)$ form a basis of $A_{*, *}$ follows now from Theorem 12.4.

Theorem 12.6 The (graded commutative) algebra $\mathcal{A}^{*, *}(k, \mathbf{Z} / l)$ over $H^{*, *}$ is canonically isomorphic to the (graded commutative) algebra with generators

$$
\begin{aligned}
& \tau_{i} \in A_{2 l^{i}-1, l^{i}-1} \\
& \xi_{i} \in A_{2 l^{i}-2, l^{i}-2}
\end{aligned}
$$

and relations

$$
\begin{aligned}
& \text { 1. } \xi_{0}=1 \\
& \text { 2. } \tau_{i}^{2}= \begin{cases}0 & \text { for } l \neq 2 \\
\tau \xi_{i+1}+\rho \tau_{i+1}+\rho \tau_{0} \xi_{i+1} & \text { for } l=2\end{cases}
\end{aligned}
$$

Proof: We already know by Corollary 12.5 that $\mathcal{A}_{*, *}(k, \mathbf{Z} / l)$ has a basis which consist of monomials in $\xi_{i}, \tau_{i}$ which are of degree $\leq 1$ in each $\tau_{i}$. The relation $\tau_{i}^{2}=0$ for odd $l$ is a corollary of graded commutativity. Thus we may assume that $l=2$ in which case we have only to show that $\tau_{i}^{2}=$ $\tau \xi_{i+1}+\rho \tau_{i+1}+\rho \tau_{0} \xi_{i+1}$. The required relation follows immediately from (12.3), the multiplicativity of $\lambda^{*, *}$ and the relation $v^{2}=\tau u+\rho v$ in the motivic cohomology ring of $B \mu_{2}$.

Lemma 12.7 For any $\phi \in A^{*, *}$ one has:

$$
\phi\left(u^{l^{j}}\right)=\sum_{i}\left\langle\xi_{i}^{l^{j}}, \phi\right\rangle u^{l^{i+j}}
$$

Proof: Let $x_{1}, x_{2}$ be any elements of $A_{*, *}$. If $\psi^{*}(\phi)=\sum \phi_{k}^{\prime} \otimes \phi_{k}^{\prime \prime}$ we have, by definition of product in $A_{*, *}$ :

$$
\left\langle x_{1} x_{2}, \phi\right\rangle=\sum\left\langle x_{1}, \phi_{k}^{\prime}\right\rangle\left\langle x_{2}, \phi_{k}^{\prime \prime}\right\rangle
$$

This implies by induction starting with (12.4) that for any $n$ one has

$$
\phi\left(u^{n}\right)=\sum_{\left(j_{1}, \ldots, j_{n}\right)}\left\langle\xi_{j_{1}} \ldots \xi_{j_{n}}, \phi\right\rangle u^{l^{j_{1}+\cdots+j_{n}}}
$$


For $n=l^{j}$ all the terms except for the ones which show up in the right hand side of (12.6) cancel out since we work with $\mathbf{Z} / l$-coefficients.

Proposition 12.8 Let $\phi, \psi$ be elements of $A^{*, *}$ such that

$$
\left\langle\xi_{k}, \psi\right\rangle,\left\langle\tau_{k}, \psi\right\rangle \in \mathbf{Z} / l \subset H^{*, *}
$$

Then one has:

$$
\begin{gathered}
\left\langle\xi_{k}, \phi \psi\right\rangle=\sum_{i}\left\langle\xi_{k-i}^{l^{i}}, \phi\right\rangle\left\langle\xi_{i}, \psi\right\rangle \\
\left\langle\tau_{k}, \phi \psi\right\rangle=\left\langle\tau_{k}, \phi\right\rangle\left\langle\xi_{0}, \psi\right\rangle+\sum_{i}\left\langle\xi_{k-i}^{l^{i}}, \phi\right\rangle\left\langle\tau_{i}, \psi\right\rangle
\end{gathered}
$$

Proof: We have by (12.4)

$$
\phi \psi(u)=\sum\left\langle\xi_{i}, \phi \psi\right\rangle u^{l^{i}}
$$

On the other hand by (12.4) and (12.6) we get:

$$
\phi \psi(u)=\phi\left(\sum\left\langle\xi_{i}, \psi\right\rangle u^{l^{i}}\right)=\sum_{i, j}\left\langle\xi_{j}^{l^{i}}, \phi\right\rangle\left\langle\xi_{i}, \psi\right\rangle u^{l^{i+j}}
$$

Comparing coefficients at powers of $u$ we get the first of the required equalities. To get the second one we write

$$
\phi \psi(v)=\left\langle\xi_{0}, \phi \psi\right\rangle v+\sum_{i}\left\langle\tau_{i}, \phi \psi\right\rangle u^{l^{i}}
$$

by (12.4). And by (12.4) and (12.6) we get:

$$
\begin{gathered}
\phi \psi(v)=\left\langle\xi_{0}, \psi\right\rangle \phi(v)+\sum_{i}\left\langle\tau_{i}, \psi\right\rangle \phi\left(u^{l^{i}}\right)= \\
=\left\langle\xi_{0}, \phi\right\rangle\left\langle\xi_{0}, \psi\right\rangle v+\sum_{i}\left\langle\tau_{i}, \phi\right\rangle\left\langle\xi_{0}, \psi\right\rangle u^{l^{i}}+\sum_{i, j}\left\langle\xi_{j}^{l^{i}}, \phi\right\rangle\left\langle\tau_{i}, \psi\right\rangle u^{l^{i+j}}
\end{gathered}
$$

Comparing coefficients we get the second equality.

Now we can describe the dual to the ring structure on $A^{*, *}$. We have two $H_{*, *}$ module structures on $A_{*, *}$. The first one, the left module structure which we used all the time, is given by

$$
a *_{l} \phi(x)=\phi(a x)=a \phi(x)
$$


where $\phi \in A_{*, *}, a \in H^{*, *}$ and $x \in A^{*, *}$. The other one is the right module structure given by

$$
\phi *_{r} a(x)=\phi(x a)
$$

Lemma 11.7 implies that $\left(\phi \phi^{\prime}\right) *_{r} a=\phi\left(\phi^{\prime} *_{r} a\right)$ and, in particular, that $\phi *_{r} a=\phi\left(1 *_{r} a\right)$. The map $a \mapsto 1 *_{r} a$ coincides with the map $\lambda^{*, *}$ for $X=\operatorname{Spec}(k)$ and we denote it by $\lambda$. Therefore, the two module structures are defined by two ring homomorphisms $a \mapsto a \xi_{0}$ and $a \mapsto \lambda(a)$ from $H^{*, *}$ to $A_{*, *}$.

Denote by $A_{*, *} \otimes_{r, l} A_{*, *}$ the tensor product with the property

$$
\left(\phi *_{r} a\right) \otimes \psi=\phi \otimes\left(a *_{l} \psi\right) .
$$

Similarly, denote by $A^{*, *} \otimes_{r, l} A^{*, *}$ the tensor product with the property

$$
x a \otimes y=x \otimes a y .
$$

The following lemma is taken from [2, Lemma 3.3].

Lemma 12.9 Let $f, g$ be elments of $A_{*, *}$ and $x, y$ elements of $A^{*, *}$. The formula:

$$
\langle\theta(f \otimes g), x \otimes y\rangle=(-1)^{\operatorname{deg}(g) \operatorname{deg}(m)}\langle f, x\langle g, y\rangle\rangle
$$

defines an isomorphism

$$
\theta: A_{*, *} \otimes_{r, l} A_{*, *} \rightarrow\left(A^{*, *} \otimes_{r, l} A^{*, *}\right)^{*}
$$

where the upper star on the right hand side denotes the bigraded dual of left $H^{*, *}$-module maps from $A^{*, *} \otimes_{H^{*, *}} A^{*, *}$ to $H^{*, *}$.

Proof: One verifies easily that $\theta$ is indeed well defined by (12.7). To prove that it is an isomorphism consider the basis $\omega(I)$ in $A_{*, *}$ and let $\omega(I)^{*}$ be the dual basis in $A^{*, *}$. The elements $\omega(I) \otimes \omega(J)$ clearly generate $A_{*, *} \otimes_{r, l} A_{*, *}$ as a left $H^{* * *}$-module. The image of $\omega(I) \otimes \omega(J)$ with respect to $\theta$ is the functional which equals one on $\omega(I)^{*} \otimes \omega(J)^{*}$ and zero on all other elements of the form $\omega\left(I^{\prime}\right)^{*} \otimes \omega\left(J^{\prime}\right)^{*}$. This implies that $\omega(I) \otimes \omega(J)$ are linearly independent in $A_{*, *} \otimes_{r, l} A_{*, *}$ and hence form a basis of this left $H^{*, *}$-module. It also implies that $\theta$ maps this basis to a basis of $\left(A^{*, *} \otimes_{H^{*, *}} A^{*, *}\right)^{*}$ and therefore $\theta$ is an isomorphism.

Composing the dual to the multiplication map $A^{*, *} \otimes A^{*, *} \rightarrow A^{*, *}$ with $\theta$ we get a map

$$
\psi_{*}: A_{*, *} \rightarrow A_{*, *} \otimes_{r, l} A_{*, *}
$$


By construction, the map $\psi_{*}$ is defined by the property that $\psi_{*}(f)=\sum f_{i}^{\prime} \otimes$ $f_{i}^{\prime \prime}$ and for any $x, y \in A^{*, *}$ one has:

$$
\sum\left\langle f_{i}^{\prime}, x\left\langle f_{i}^{\prime \prime}, y\right\rangle\right\rangle=\langle f, x y\rangle
$$

Lemma 12.10 The map (12.8) is a ring homomorphism.

Proof: It follows from a direct computation and Lemma 11.7.

In view of Lemma 12.10 and Theorem 12.6 , the map $\psi_{*}$ is completely determined by its values on the generators $\xi_{i}, \tau_{i}$.

Lemma 12.11 One has:

$$
\begin{gathered}
\psi_{*}\left(\xi_{k}\right)=\sum_{i=0}^{k} \xi_{k-i}^{l^{i}} \otimes \xi_{i} \\
\psi_{*}\left(\tau_{k}\right)=\sum_{i=0}^{k} \xi_{k-i}^{l^{i}} \otimes \tau_{i}+\tau_{k} \otimes 1
\end{gathered}
$$

Proof: Follows from Proposition 12.8 and the formula (12.9).

Remark 12.12 The rings $H^{*, *}$ and $A_{*, *}$, two homomorphisms $H^{*, *} \rightarrow A_{*, *}$, the homomorphism $A_{*, *} \rightarrow H^{*, *}$ which takes $\tau_{i}$ for $i \geq 0$ and $\xi_{i}$ for $i>0$ to zero and the homomorphism $\psi_{*}$ form together a Hopf algebroid $\mathcal{H}(k, \mathbf{Z} / l)$. We can not give its complete description because we do not know the structure of $H^{*, *}$ and the explicit form of the homomorphism $\lambda$ which involves the action of the reduced power operations and the Bockstein in $H^{*, *}$. One can easily see however that these are the only two pieces of information missing. In the case when $l>2$ we have a coaction of the topological dual Steenrod algebra $A_{*}(l)$ (given the second grading in the way explained above) on $H^{*, *}$ and $\mathcal{H}(k, \mathbf{Z} / l)$ is the twisted product of $A_{*}(l)$ and $H^{*, *}$ with respect to this coaction. For $l=2$ consider the Hopf algebroid $\mathcal{H}(2)$ over $\mathbf{Z} / 2$ defined as follows:

Ring of objects is $\mathbf{Z} / 2[\rho, \tau]$ where $\operatorname{deg}(\rho)=(-1,-1)$ and $\operatorname{deg}(\tau)=(-1,0)$

Ring of morphisms is

$$
\mathbf{Z} / 2\left[\rho, \tau, \tau_{i}, \xi_{i+1}\right]_{i \geq 0} /\left(\tau_{i}^{2}-\tau \xi_{i+1}-\rho \tau_{i+1}-\rho \tau_{0} \xi_{i+1}\right)_{i \geq 0}
$$


Coface maps are given by

$$
\begin{gathered}
d_{0}(\rho)=\rho \quad d_{0}(\tau)=\tau \\
d_{1}(\rho)=\rho \quad d_{1}(\tau)=\tau+\rho \tau_{0}
\end{gathered}
$$

Codegeneracy map is given by

$$
\begin{array}{ll}
s_{0}(\rho)=\rho & s_{0}(\tau)=\tau \\
s_{0}\left(\tau_{i}\right)=0 & \text { for } i \geq 0 \\
s_{0}\left(\xi_{i}\right)=0 & \text { for } i>0
\end{array}
$$

Co-composition is given by

$$
\begin{gathered}
\psi_{*}(\rho)=\rho \otimes 1=1 \otimes \rho \\
\psi_{*}(\tau)=\tau \otimes 1=1 \otimes \tau+\rho \tau_{0} \otimes 1 \\
\psi_{*}\left(\xi_{k}\right)=\sum_{i=0}^{k} \xi_{k-i}^{l^{i}} \otimes \xi_{i} \\
\psi_{*}\left(\tau_{k}\right)=\sum_{i=0}^{k} \xi_{k-i}^{l^{i}} \otimes \tau_{i}+\tau_{k} \otimes 1
\end{gathered}
$$

Note that our formulas imply that $\mathcal{H}(2)$ is, in fact, a Hopf algebroid over $\mathbf{Z} / 2[\rho]$. This Hopf algebroid co-acts on $H^{*, *}$ and $\mathcal{H}(k, \mathbf{Z} / 2)$ is the twisted product of $\mathcal{H}(2)$ and $H^{*, *}$ with respect to this coaction.

\section{Operations $\rho(E, R)$ and their properties}

Let $R=\left(r_{1}, r_{2}, \ldots\right)$ be a sequence of non-negative integers which are almost all zero and $E=\left(\epsilon_{0}, \epsilon_{1}, \ldots\right)$ a sequence of zeros and ones which are almost all zeros. Corollary 12.5 implies that elements of the form

$$
\tau(E) \xi(R):=\prod_{i \geq 0} \tau_{i}^{\epsilon_{i}} \prod_{j \geq 1} \xi_{j}^{r_{j}}
$$

form a basis of $A_{*, *}$ over $H^{*, *}$. Let $\rho(E, R)$ be the dual basis of $A^{*, *}$. In particular, we define

$$
\mathcal{P}^{R}=\rho(\underline{0}, R)
$$




$$
Q(E):=\rho(E, \underline{0})
$$

and

$$
Q_{i}=Q(0, \ldots, 0,1,0, \ldots)
$$

where 1 is on the i-th place i.e. $Q_{i}$ is the dual to $\tau_{i}$.

If $l$ is odd operations $\rho(E, R)$ and, in particular, $Q(E), \mathcal{P}^{R}$ and $Q_{i}$, have the same properties as their topological counterparts defined in [5]. In what follows we assume that $l=2$.

Lemma $13.1 \mathcal{P}^{(n, 0, \ldots, 0, \ldots)}=P^{n}$

Proof: Follows immediately from Theorem 12.6.

Proposition $13.2 \rho(E, R)=Q(E) \mathcal{P}^{R}$.

Proof: We have to compute the pairing of $Q(E) \mathcal{P}^{R}$ with $\tau\left(E^{\prime}\right) \xi\left(R^{\prime}\right)$ and show that it is 1 for $E^{\prime}=E, R^{\prime}=R$ and zero otherwise. By (12.9) we have:

$$
\left\langle\tau\left(E^{\prime}\right) \xi\left(R^{\prime}\right), Q(E) \mathcal{P}^{R}\right\rangle=\sum\left\langle f_{i}^{\prime}, Q(E)\left\langle f_{i}^{\prime \prime}, \mathcal{P}^{R}\right\rangle\right\rangle
$$

where

$$
\sum f_{i}^{\prime} \otimes f_{i}^{\prime \prime}=\psi_{*}\left(\tau\left(E^{\prime}\right) \xi\left(R^{\prime}\right)\right)=\psi_{*}\left(\tau\left(E^{\prime}\right)\right) \psi_{*}\left(\xi\left(R^{\prime}\right)\right)
$$

We can choose our representation $\sum f_{i}^{\prime} \otimes f_{i}^{\prime \prime}$ such that $f_{i}^{\prime \prime}$ are of the form $\rho\left(E^{\prime \prime}, R^{\prime \prime}\right)$ and, in particular, $\left\langle f_{i}^{\prime \prime}, \mathcal{P}^{R}\right\rangle$ are in $\mathbf{Z} / 2$. Then, the expression (13.1) depends only on the class of $\psi_{*}\left(\tau\left(E^{\prime}\right) \xi\left(R^{\prime}\right)\right)$ in $A_{*, *} / I \otimes_{r, l} A^{*, *}$ where $I$ is the ideal generated by $\xi_{i}$ for $i>0$. In this quotient ring we have:

$$
\begin{gathered}
\psi_{*}\left(\xi_{k}\right)=1 \otimes \xi_{k} \\
\psi_{*}\left(\tau_{k}\right)=1 \otimes \tau_{k}+\tau_{k} \otimes 1
\end{gathered}
$$

This easily implies that (13.1) is non-zero if and only if $E=E^{\prime}$ and $R=R^{\prime}$.

Let $\rho$ be, as before, the class of -1 in $H^{1,1}$. Denote by $B^{*, *}$ the $\mathbf{Z} / 2[\rho]$ submodule in $A^{*, *}$ generated by elements of the form $Q(E)$. Let $B_{*, *}$ be the dual of $B^{*, *}$. Then

$$
B_{*, *} \otimes_{\mathbf{z} / 2[\rho]} H^{*, *}=A_{*, *} /\left(\left\{\xi_{i}\right\}\right) .
$$


Lemma 12.11 implies that for $f \in I=\left(\left\{\xi_{i}\right\}\right)$, one has

$$
\psi_{*}(f) \in I \otimes A_{*, *}+A_{*, *} \otimes I
$$

and, therefore, $\psi_{*}$ defines a comultiplication on $B_{*, *} \otimes_{\mathbf{z} / 2[\rho]} H^{*, *}$ which takes $\tau_{k}$ to $\tau_{k} \otimes 1+1 \otimes \tau_{k}$. From this one easily gets the following result.

Proposition 13.3 As a $\mathbf{Z} / 2[\rho]$-algebra, $B_{*, *}$ is of the form

$$
B_{*, *}=\mathbf{Z} / 2[\rho]\left[\tau_{0}, \ldots, \tau_{n}, \ldots\right] /\left(\tau_{i}^{2}=\rho \tau_{i+1}\right)
$$

The map $\psi_{*}$ defines a Hopf algebra structure on $B_{*, *}$ over $\mathbf{Z} / 2[\rho]$, satisfying:

$$
\phi_{*}\left(\tau_{i}\right)=\tau_{i} \otimes 1+1 \otimes \tau_{i} .
$$

Dualizing we get the following theorem on the structure of $B^{*, *}$.

Proposition 13.4 As a $\mathbf{Z} / 2[\rho]$-algebra, $B^{*, *}$ is the exterior algebra with generators $Q_{i}$. For $E=\left(\epsilon_{0}, \ldots, \epsilon_{n}\right)$ one has $Q(E)=\prod Q_{i}^{\epsilon_{i}}$. The coproduct is given on $Q_{i}$ 's by

$$
\psi^{*}\left(Q_{i}\right)=1 \otimes Q_{i}+Q_{i} \otimes 1+\rho \sum_{E, E^{\prime}} c_{E, E^{\prime}} Q(E) \otimes Q\left(E^{\prime}\right)
$$

where $E, E^{\prime}$ run through sequences of zeros and ones which are almost all zeros and $c_{E, E^{\prime}}$ are elements of $H^{*, *}$.

The following three results complete the proof of all the properties of operations $Q_{i}$ used in [9].

Lemma $13.5 Q_{0}=\beta$.

Proof: Since operations $\rho(E, R)$ form a basis we can write $\beta$ as a formal linear combination $\sum a_{E, R} \rho(E, R)$. Since the weight of $\beta$ is zero this implies that $\beta=c Q_{0}$ for $c \in \mathbf{Z} / l$. Since $\beta(u)=v$, formula (12.4) implies that $c=1$.

Proposition 13.6 Let $i \geq 1$ and $q_{i}=\mathcal{P}^{0, \ldots, 0,1,0, \ldots}$ be the dual to $\xi_{i}$. Then one has

$$
Q_{i}=\left[Q_{0}, q_{i}\right]
$$


Proof: We have to show that $q_{i} Q_{0}=Q_{0} q_{i}+Q_{i}$ i.e. that the only monomials which pair non-trivially with $q_{i} Q_{0}$ are $\tau_{0} \xi_{i}$ and $\xi_{i}$ and that for those monomials the pairing gives 1 . Using formula (12.9) we see that it is sufficient to show that the only monomials $M=\tau(E) \xi(R)$ such that $\xi_{i} \otimes \tau_{0}$ appears in the decomposition of $\psi_{*}(M)$ are $\tau_{0} \xi_{i}$ and $\tau_{i}$ and that for those monomials $\xi_{i} \otimes \tau_{0}$ appears with coefficient 1 . The later follows immediately from Lemma 12.11. To prove the former note that the question of whether or not $\xi_{i} \otimes \tau_{0}$ appears in the expression for $\psi_{*}(M)$ depend only on the class of $\psi_{*}(M)$ in $A_{*, *} \otimes_{r, l} A_{*, *} / J$ where $J$ is generated by $\tau_{k}$ for $k>0$. In this quotient $\psi_{*}\left(\tau_{k}\right)=\xi_{k} \otimes \tau_{0}+\tau_{k} \otimes 1$ and $\tau_{0}^{2}=\tau \xi_{1}+\rho \tau_{0} \xi_{1}$. This shows that the only way to get $\xi_{i} \otimes \tau_{0}$ is to consider $\psi_{*}\left(\tau_{i}\right)$ or $\psi_{*}\left(\tau_{0} \xi_{i}\right)$.

The following example shows that not all of the standard topological formulas for $Q_{i}$ 's hold in the motivic context.

Example 13.7 In topology, one can define operations $Q_{i}$ in terms of the Steenrod squares inductively by the formula $Q_{0}=S q^{1}, Q_{i+1}=\left[Q_{i}, S q^{2^{i+1}}\right]$. Let us show that in the motivic Steenrod algebra $Q_{2} \neq\left[Q_{1}, S q^{4}\right]$ if $\rho \neq 0$ i.e. if $k$ does not contain the square root of -1 . Using (12.9) and Lemma 12.11 we can compute $S q^{4} Q_{1}$ in terms of the basis dual to $\tau(E) \xi(R)$. We get:

$$
S q^{4} Q_{1}-Q_{1} S q^{4}=Q_{2}+\rho Q_{0} Q_{1} S q^{2}
$$

\section{Operations and characteristic classes}

The goal of this section is to prove Theorem 14.2. For a smooth scheme $X$, let $K_{0}(X)$ be the Grothendieck group of vector bundles on $X$. All schemes in this section are assumed to be quasi-projective.

Theorem 14.1 For any symmetric polynomial $s=s\left(t_{1}, \ldots, t_{n}, \ldots\right)$ there exists a unique natural transformation of contravariant functors from smooth quasi-projective varieties to pointed sets of the form:

$$
c_{s}: K_{0}(-) \rightarrow \oplus_{n \geq 0} H^{2 n, n}(-, \mathbf{Z})
$$

such that for a collection of line bundles $L_{1}, \ldots, L_{n}$ on $X$ one has

$$
c_{s}\left(\oplus_{i=1}^{n} L_{i}\right)=s\left(e\left(L_{1}\right), \ldots, e\left(L_{n}\right)\right)
$$


Proof: It follows in the standard way from Theorem 4.1.

Let $\phi \in A^{p, q}$ be a cohomological operation. For any $X$ and a vector bundle $V$ on $X$ the value of $\phi$ on the Thom class $t_{V}$ is, by Proposition 4.3, of the form $c_{\phi}(V) t_{V}$ where $c_{\phi}(V)$ is a well defined class in $H^{p, q}(X, \mathbf{Z} / l)$.

Theorem 14.2 Let $E=\left(\epsilon_{0}, \ldots, \epsilon_{d}\right), R=\left(r_{1}, \ldots, r_{n}\right)$ be as in Section 13. Then for a vector bundle $V$ one has:

1. $c_{\rho(E, R)}(V)=0$ if $E \neq 0$

2. $c_{\mathcal{P}(R)}(V)=s_{R}(V)$ where $s_{R}$ is the reduction modulo $l$ of the characteristic class corresponding by Theorem 14.1 to the symmetric polynomial

$$
\sum_{f} \prod_{j \in\{0, \ldots, n\}}\left(\prod_{i \in f^{-1}(j)} t_{i}\right)^{l^{j}-1}
$$

where $f$ runs through all functions $\{1, \ldots, m, \ldots\} \rightarrow\{0, \ldots, n\}$ such that for any $i, n \geq i>0$ one has $\left|f^{-1}(i)\right|=r_{i}$.

Corollary 14.3 Let $q_{n}=\mathcal{P}(0, \ldots, 0,1,0, \ldots)$ be the operation dual to $\xi_{n}$. Then one has

$$
q_{n}\left(t_{V}\right)=s_{l^{n}-1}(V) t_{V}
$$

where $s_{j}$ is the characteristic class corresponding, by Theorem 14.1, to the symmetric function $\sum t_{i}^{j}$.

Corollary 14.4 One has

$$
P^{n}\left(t_{V}\right)=c_{n, l-1}(V) t_{V}
$$

where $c_{n, j}$ is the characteristic class corresponding, by Theorem 14.1, to the symmetric function $\sum t_{i_{1}}^{j} \ldots t_{i_{n}}^{j}$.

The proof of this theorem occupies the rest of this section.

Lemma 14.5 Let $X$ be a smooth scheme and $w$ an element in $H^{2,1}(X, \mathbf{Z} / l)$ which is the reduction modulo $l$ of an integral class. Then there exists a map $f: X_{+} \rightarrow\left(B \mu_{l}\right)_{+}$in $H_{\text {. such that } w}=f^{*}(v)$. 
Proof: Since $X$ is quasi-projective the Jouanolou trick (see []) shows that there exist an affine scheme $X^{\prime}$ and an $\mathbf{A}^{1}$-weak equivalence $X^{\prime} \rightarrow X$. Therefore, we may assume that $X$ is affine. By [][] and Lemma 4.5, any element of $H^{2,1}(X, \mathbf{Z})$ is of the form $e(L)$ for a line bundle $L$. Since $X$ is affine there is a map $g: X \rightarrow \mathbf{P}^{N}$ for some $N$ such that $L=g^{*}(\mathcal{O}(1))$. On the other hand the reduction of $e(\mathcal{O}(1))$ modulo $l$ is $p^{*}(v)$ where $p: \mathbf{P}^{N} \rightarrow B \mu_{l}$ is the standard morphism. This proves the lemma.

Lemma 14.6 Let $X$ be a smooth scheme and $w$ an element in $H^{2,1}(X, \mathbf{Z} / l)$ which is the reduction modulo $l$ of an integral class. Let further $\phi$ be an operation of the form $\rho(E, R)$. Then one has

$$
\phi(w)= \begin{cases}w^{l^{n}} & \text { if } \phi=q_{n} \\ 0 & \text { otherwise }\end{cases}
$$

Proof: By Lemma 14.5 it is sufficient to prove our statement for $X=B \mu_{l}$ and $w=v$. In this case our result follows from (12.5).

Lemma 14.7 Let $L$ be a line bundle and $\sigma$ the class of $\mathcal{O}(-1)$ in $H^{* * *}(\mathbf{P}(L \oplus$ $\mathcal{O}), \mathbf{Z})$. Then one has $\sigma^{2}=-e(L) \sigma$.

Proof: Using standard argument we can reduce the problem to the case $X=\mathbf{P}^{N}$ and $L=\mathcal{O}(1)$. The restriction of $\sigma$ to $\mathbf{P}(\mathcal{O})$ is zero. Hence, $\sigma^{2}=x \sigma$ for some $x$. The restriction of $\sigma$ to $\mathbf{P}(L)$ is $-e(L)$. Hence $-e(L) x=e(L)^{2}$. Since $e(\mathcal{O}(1))$ is not a zero divisor, we conclude that $x=-e(L)$.

Lemma 14.8 Let $L$ be a line bundle and $\phi$ an operation of the form $\rho(E, R)$. Then one has

$$
\phi\left(t_{L}\right)= \begin{cases}e(L)^{l^{n}-1} t_{L} & \text { for } \phi=q_{n} \\ 0 & \text { otherwise }\end{cases}
$$

Proof: Consider the standard projection $p: \mathbf{P}(\mathcal{O} \oplus L) \rightarrow T h(L)$. As shown in Section 4, it defines a monomorphism on motivic cohomology. Together with Lemma 14.6 this immediately implies that $\phi\left(t_{L}\right)=0$ if $\phi \neq q_{n}$ for some $n$. As shown in the proof of Lemma 4.5 we have $p^{*}\left(t_{L}\right)=\sigma+e(L)$. Hence, by Lemma 14.6 and Lemma 14.7 we have

$$
p^{*} q_{n}\left(t_{L}\right)=q_{n} p^{*}\left(t_{L}\right)=(\sigma+e(L))^{l^{n}}=e(L)^{l^{n}-1}(\sigma+e(L))
$$

Since $p^{*}$ is a monomorphism we conclude that $q_{n}\left(t_{L}\right)=e(L)^{l^{n}-1} t_{L}$. 
Remark 14.9 Lemma 14.8 has the following analog for the basis of admissible monomials instead of Milnor's basis $\rho(E, R)$. Recall, that $M_{k}$ denotes the monomial $P^{l^{k-1}} \ldots P^{l} P^{1}$. If $L$ is a line bundle and $\phi$ an admissible monomial then one has

$$
\phi\left(t_{L}\right)= \begin{cases}e(L)^{l^{k}-1} t_{L} & \text { for } \phi=M_{k} \\ 0 & \text { otherwise }\end{cases}
$$

Let $I$ be the two-sided ideal of $A^{*, *}$ generated by $Q_{0}=\beta$. Proposition 13.6 implies that it coincides with the two-sided ideal generated by $Q(E)$ for $E \neq$ 0 . Since $\psi^{*}(\beta)=\beta \otimes 1+1 \otimes \beta$ for any $\phi \in I$ we have $\psi^{*}(\phi) \in A^{* * *} \otimes I+I \otimes A^{*, *}$. In particular, if $w, w^{\prime}$ are motivic cohomology classes such that $\phi(w)=0$, $\phi\left(w^{\prime}\right)=0$ for any $\phi \in I$ then $\phi\left(w w^{\prime}\right)=0$ for any $\phi \in I$. Together with the splitting principle and Lemma 14.8 this implies the following result.

Lemma 14.10 For any $E \neq 0$ and any vector bundle $V$ one has $c_{\rho(E, R)}(V)=$ 0 .

Let $R=\left(r_{1}, \ldots, r_{n}\right)$ be a sequence of non-negative integers. To prove the second statement of Theorem 14.2 we have to compute $\mathcal{P}(R)\left(t_{L_{1}} \wedge t_{L_{m}}\right)$ for a collection of line bundles $L_{1}, \ldots, L_{m}$. Let $\psi_{m}^{*}$ be the m-fold iteration of the comultiplication map for $A^{*, *}$ and

$$
\psi_{m}^{*}(\mathcal{P}(R))=\sum a_{\left(E_{1}, R_{1}, \ldots, E_{m}, R_{m}\right)} \rho\left(E_{1}, R_{1}\right) \otimes \ldots \otimes \rho\left(E_{m}, R_{m}\right)
$$

By Lemma 14.8 we have

$$
\mathcal{P}(R)\left(t_{L_{1}} \wedge \cdots \wedge t_{L_{m}}\right)=\left(\sum a_{\left(R_{1}, \ldots, R_{m}\right)} \prod_{i=1}^{m} c_{\mathcal{P}\left(R_{i}\right)}\left(L_{i}\right)\right) \wedge t_{L_{1}} \wedge \cdots \wedge t_{L_{m}}
$$

where the only non-trivial terms are those for which $R_{i}$ is of the form

$$
R_{i}=(0, \ldots, 0,1,0, \ldots)
$$

On the other hand we have

$$
\begin{aligned}
& a_{\left(R_{1}, \ldots, R_{m}\right)}=\left\langle\psi_{m}^{*}(\mathcal{P}(R)), \xi\left(R_{1}\right) \otimes \ldots \otimes \xi\left(R_{m}\right)\right\rangle= \\
& =\left\langle\mathcal{P}(R), \xi\left(R_{1}\right) \ldots \xi\left(R_{n}\right)= \begin{cases}1 & \text { if } \sum R_{i}=R \\
0 & \text { otherwise }\end{cases} \right.
\end{aligned}
$$

A sequence of $R_{i}$ 's of the form $(0, \ldots, 0,1,0, \ldots)$ can be thought of as a function $f: i \rightarrow f(i)$ such that $R_{i}=q_{f(i)}$ where $q_{0}$ is assumed to be 1 . The condition $\sum R_{i}=R$ means that we consider the functions which take the value $i>0$ exactly $r_{i}$ times. Together with the fact that $c_{q_{n}}(L)=e(L)^{l^{n}-1}$ this proves the last statement of Theorem 14.2. 


\section{References}

[1] S. Bloch. The moving lemma for higher Chow groups. J. Algebr. Geom., 3(3):537-568, Feb. 1994.

[2] J. M. Boardman. The eightfold way to BP-operations. In Current trends in algebraic topology, pages 187-226. AMS/CMS, Providence, 1982.

[3] Pierre Deligne. Voevodsky's lectures on motivic cohomology. www.math.ias.edu/vladimir/seminar.html, 2000/2001.

[4] J. Milnor. Algebraic K-theory and quadratic forms. Inv. Math., 9:318344, 1970.

[5] John Milnor. The Steenrod algebra and its dual. Annals of Math., 67(1):150-171, 1958.

[6] Fabien Morel and Vladimir Voevodsky. $\mathbf{A}^{1}$-homotopy theory of schemes. Publ. Math. IHES, (90):45-143, 1999.

[7] N. E. Steenrod and D. B. Epstein. Cohomology operations. Princeton Univ. Press, Princeton, 1962.

[8] Andrei Suslin and Vladimir Voevodsky. Bloch-Kato conjecture and motivic cohomology with finite coefficients. In The arithmetic and geometry of algebraic cycles, pages 117-189. Kluwer, 2000.

[9] Vladimir Voevodsky. The Milnor Conjecture. www.math.uiuc.edu/Ktheory/170, 1996.

[10] Vladimir Voevodsky. Motivic cohomology are isomorphic to higher Chow groups. www.math.uiuc.edu/K-theory/378, 1999.

[11] Vladimir Voevodsky. The Milnor Conjecture (2001). Preprint, 2001. 